Published in Journal of Abnormal Psychology, 130(5), 443-454. (c) 2021, American Psychological Association. This paper is not the copy of record and may not exactly replicate the final, authoritative version of the article. Please do not copy or cite without authors' permission. The final article will be available, upon publication, via its DOI: 10.1037/abn0000681

\title{
A Computational Account of the Mechanisms Underlying Face Perception Biases in Depression
}

\author{
Fabian A. Soto \\ Department of Psychology \\ Florida International University \\ Sanaz Hosseini, Jason Hays \\ Department of Psychology \\ Florida International University
}

\author{
Rochelle A. Stewart \\ Department of Psychology \\ University of Texas at Austin \\ Christopher G. Beevers \\ Department of Psychology \\ University of Texas at Austin
}

\begin{abstract}
Here, we take a computational approach to understand the mechanisms underlying face perception biases in depression. Thirty participants diagnosed with Major Depressive Disorder and thirty healthy control participants took part in three studies involving recognition of identity and emotion in faces. We used signal detection theory to determine whether any perceptual biases exist in depression aside from decisional biases. We found lower sensitivity to happiness in general, and lower sensitivity to both happiness and sadness with ambiguous stimuli. Our use of highly-controlled face stimuli ensures that such asymmetry is truly perceptual in nature, rather than the result of studying expressions with inherently different discriminability. We found no systematic effect of depression on the perceptual interactions between face expression and identity. We also found that decisional strategies used in our task were different for people with depression and controls, but in a way that was highly specific to the stimulus set presented. We show through simulation that the observed perceptual effects, as well as other biases found in the literature, can be explained by a computational model in which channels encoding positive expressions are selectively suppressed.
\end{abstract}

Keywords: depression, face perception, perceptual bias, computational modeling

Cognitive models of depression suggest that the development and maintenance of this disorder stem from individuals' characteristic ways of attending to, interpreting, and remembering stimuli in their environment, such as selective attention towards negative aspects of experience or interpreting objectively ambiguous information as negative (Beck, 2008; Disner et al., 2011). Biased attention and cognition have been associated with sustained negative affect (e.g., Bar-Haim et al., 2007; Peckham et al., 2010), affective psychopathology (e.g., Bar-Haim et al., 2010; Wilkinson and Goodyer, 2006) and predict future development of depression (e.g., Abela and Hankin, 2011; Beevers et al., 2011). Thus, there is considerable support for cognitive models of depression (for reviews, see Disner et al., 2011; Gotlib and Joormann, 2010).

One of the ways in which biases are expressed in depression is in the processing of face emotion. In-depth reviews and meta-analyses of the literature (e.g., Bistricky et al., 2011; Bourke et al., 2010) have concluded that people with depression show a bias toward interpreting ambiguous faces (e.g., neutral or morphed) as expressing negative emotion and a general impairment in processing of emotional faces. Interpersonal theories of depression posit that depressed indi- viduals are particularly alert for signs of interpersonal rejection or negative feedback in an effort to reduce social rejection (Joiner and Metalsky, 1995; Surguladze et al., 2004). Indeed, depression is commonly accompanied by impairments in social functioning (Bistricky et al., 2011; Surguladze et al., 2004). Biased processing of expression information might be a key mechanism producing or exacerbating such impairments, as decoding facial expression correctly is critical for adequate social interaction (Leppänen and Hietanen, 2001; Marsh et al., 2007).

Currently, there is little understanding of the mechanisms underlying face processing biases in depression. A first important question is whether or not there are any perceptual mechanisms underlying such biases (i.e., people with depression perceiving emotional expression differently), aside from any decisional or cognitive mechanisms (i.e., people with depression interpreting emotional expression differently). Because there is evidence of higher-level biases in depression, the simplest explanation is that face processing biases are decisional in nature. Whether biases are perceptual or decisional has an impact on how they should be addressed in treatment. Reducing perceptual biases is likely to require extensive feedback-based training like that provided by atten- 
tional bias modification (ABM; Bar-Haim, 2010; MacLeod, 2012), aimed at inducing perceptual and attentional effects. Decisional biases can be manipulated through short training interventions and verbal instructions (Ashby et al., 2001), and might not require special treatment beyond traditional therapy.

Similarly, whether or not depression affects face perception could have an impact on how generalizable the results of ABM and other forms of feedback learning are. Generalization of learning increases when task-relevant stimulus features are "separable" from irrelevant stimulus features (Garner, 1974; Goldstone, 1994); separable features are those that can be selectively attended and processed independently from one another.

In $\mathrm{ABM}$, the relevant task features are those related to emotional expression of faces, whereas the most common irrelevant face features are those related to face identity. In healthy participants, learning involving such face dimensions generalizes well, similarly to more traditional separable dimensions (Soto and Ashby, 2019). A deficit in the separability of emotion from identity would impair generalization of ABM-induced learning to new faces. The sum of our current knowledge about this issue comes from a single study by Gilboa-Schechtman et al. (2004), which found that depressed participants had more trouble than controls ignoring emotional expression while processing face identity. Again, it is not clear whether such results were due to perceptual versus decisional factors.

\section{Dissociating perceptual versus decisional mechanisms of bias}

The most influential framework used to dissociate the contribution of perceptual and decisional processes in perceptual tasks is signal detection theory (SDT; Green and Swets, 1966). Imagine that the task of an individual is simply to indicate whether the faces shown in Figure 1a show a sad expression. According to SDT, this process involves two steps. First, the perceptual system extracts sensory evidence that the face is showing a sad expression, as represented by the "Perception" box in Figure 1a. This process is influenced by perceptual noise, so the presentation of the same face in different occasions should lead to different levels of sensory evidence. Such noisy representations are depicted in Figure 1a by two normal distributions, one for the neutral face (red) and one for the sad face (blue). In this example both distributions have a standard deviation equal to one, so the distance between their means represents $d^{\prime}$, a commonly-used measure of perceptual discriminability. Second, the observer sets up a decision bound, represented by the "Decision" box in Figure 1a. This is a threshold indicating how much sensory evidence is required to decide that the face is showing a sad expression. The lower this bound, the more bias exists to indicate that the face is sad. SDT provides ways to separately quantify these perceptual and decisional factors.

In the traditional analysis of a discrimination task, an overall proportion of correct responses is computed from the trials in which the participant correctly identifies face emotion. SDT can dissociate between perceptual and decisional factors because it uses more detailed information about patterns of errors. For example, moving the threshold in Figure 1a to the right would result in more correct identifications of neutral faces (the participant would change a bias to respond "sad" for a bias to respond "neutral"), but at the cost of more errors in the identification of sad faces. The full pattern of correct responses and errors, together with the constraints imposed by the model (e.g., the assumption of normally distributed noise) allow to independently infer d' and bias from data.

The extension of SDT to multiple perceptual dimensions, known as general recognition theory (GRT; for a review see Ashby and Soto, 2015), allows researchers to study how different stimulus components interact during processing. According to GRT, processing of one face property can affect processing of a second face property both at the perceptual and decisional levels. Figure $1 \mathrm{~b}$ shows an example. As before, imagine that the task is to detect sadness in a face. However, now the face will also vary in identity. In Figure $1 \mathrm{~b}$, the top faces correspond to one identity, and the bottom faces correspond to another (although they might look very similar to some readers). As in SDT, emotion processing of each face involves a perceptual stage, in which the stimulus produces a noisy representation of evidence for sadness, and a decision stage, in which a threshold is used to determine whether the evidence is high enough to report sadness in the face.

As shown in Figure 1b, the processing of identity in the faces can interact with emotion processing in two ways. The first way is perceptual: changes in identity could affect how face emotion is perceived. For example, focus on the two blue distributions, representing stimuli showing sadness. The top identity is represented by a distribution drawn with a solid line, and the bottom identity by a distribution drawn with a dotted line. The area between the two distributions, shaded in blue, is a measure of their distance commonly known as $L 1$ distance. It measures to what extent changes in identity produce changes in perceptual representations of sad faces. Similarly, it is possible to measure the $L 1$ distance between the two faces showing a neutral expression (area shaded in red). Note how in this example the actual discriminability of emotion, measured through $d^{\prime}$ (i.e., the distance between the solid red and blue distributions, and between the dotted red and blue distributions), is the same across identities. However, identity clearly influences perception of sadness. In GRT, this is known as a violation of perceptual separability, and it is different from discriminability. 
a. SDT dissociates perceptual and decisional effects on discrimination performance

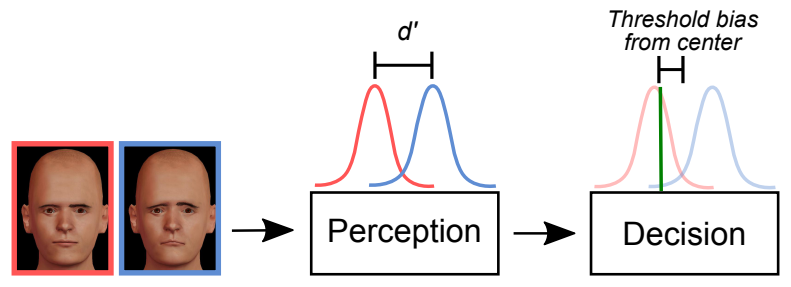

b. GRT dissociates perceptual and decisional mechanisms of interaction between dimensions

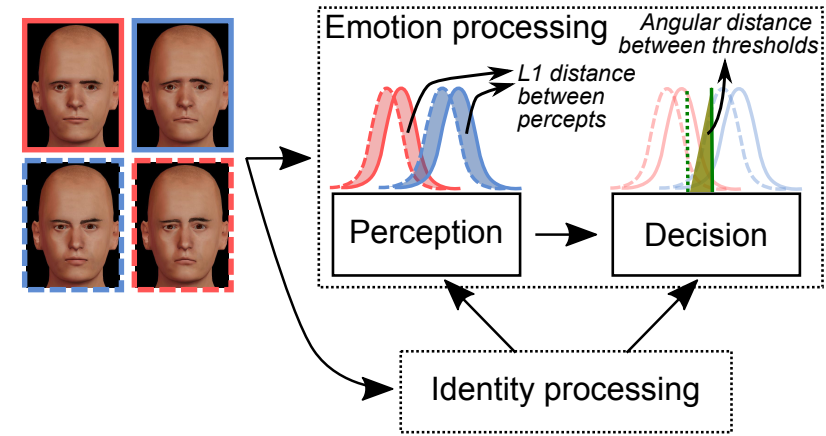

Figure 1. Schematic explanation of the assumptions behind SDT (a) and GRT (b).

The second way in which processing of identity and emotion can interact is at the level of decision making, as shown in the right part of Figure 1b. The threshold used to make decisions about sadness might change depending on face identity. This is represented by two different thresholds in Figure $1 \mathrm{~b}$, one to determine sadness in the top identity (solid green) and the other to determine sadness in the bottom identity (dotted green). In GRT, this interaction is known as a violation of decisional separability, and it can be measured by the distance between bounds (angular distance is preferred due to the way in which the model is implemented).

To the best of our knowledge, only one study in children has used SDT to dissociate perceptual and decisional contributions to face biases in depression (Schepman et al., 2012), although other researchers have attempted the dissociation through other means (Gilboa-Schechtman et al., 2004; Surguladze et al., 2004). Clearly, there is a lack of research determining whether depression produces biases in the perception of facial expression, beyond the cognitive and decisional biases that it produces.

\section{The current study}

Here, we use SDT to dissociate perceptual and decisional mechanisms underlying face processing biases in depression, by performing a model-based analysis of data from a face identification task. The task involves four stimuli, which result from the combination of two face properties (identity and emotion) with two levels each (e.g., Joe vs. Bob, Neutral vs. $\mathrm{Sad})$. In each trial of this simple task, a stimulus is presented and it must be identified through a specific response. The stimuli were made confusable through morphing. The pattern of confusion errors was fitted to a version of GRT (Soto et al., 2015), and measures of sensitivity, bias, and perceptual and decisional separability were computed directly from the estimated model parameters and compared between groups.

Participants were presented with three different identification tasks, involving different combinations of emotions: neutral vs. sad, neutral vs. happy, and sad vs. happy. To determine whether any results obtained with one set of stimuli would generalize to a different stimulus set, we performed a second session on a different day using stimuli obtained from a second pair of face identities. The stimuli were carefully controlled so that the discriminability of identity and expression was comparable across stimulus sets.

Previous research shows that when ambiguity is experimentally increased, biases observed in depression become stronger (Schepman et al., 2012). Thus, the study included a final session on a third day, which was identical to the first session but included stimuli that were made ambiguous by increasing similarity in both the identity and emotion dimensions.

Because the intensity of the expression itself can reveal or obscure any deficits shown by depression in emotion recognition, it was important to equate the intensity of the sadness and happiness expressions. This is not a common step in previous research (for a review, see Bourke et al., 2010), but we deemed it necessary to obtain data which could constrain potential mechanisms underlying the observed biases (e.g., the specificity of the bias to a particular expression). We obtained data from a pilot study on the discriminability of different levels of expression against neutral, and chose levels of sadness and happiness with similar discriminability.

\section{Method}

\section{Participants}

Sixty adult participants from the Austin, TX area were recruited for this study, half of them $(n=30)$ in the MDD (Major Depressive Disorder) group and the other half $(n=30)$ in the Control group. No participants were included in either group that had current use of psychoactive drugs, steroidal or psychotropic medications, serious medical complications (e.g., cancer, diabetes, epilepsy or head trauma), heavy tobacco use defined as smoking 20 cigarettes per day or $>20$ pack per year, recent heavy alcohol use defined as 5 or more drinks on the same occasion on each of 5 or more days in the past 30 days, or were at imminent risk of self-harm or harm to others or having a recent history of suicidal behavior (ei- 
ther a Columbia-Suicide Severity Rating Scale score of Type 4 or 5 or suicidal behavior in the past 2 months). We did not administer a drug test to confirm the participants' self-report.

Participants in the MDD group had mean age of 23.33 (range 18-32) and 70\% $(\mathrm{n}=21)$ were female. Self-reported ancestry was as follows: $53 \%(\mathrm{n}=16)$ were European, $23 \%$ $(n=7)$ were Asian, $13 \%(n=4)$ were African-American or Black, 3\% ( $\mathrm{n}=1)$ were American Indian or Alaska Native, $13 \%(n=4)$ were more than one race or reported that none of the categories were applicable and $47 \%(\mathrm{n}=14)$ were Hispanic. They were screened to have a score of 11 or greater in the Quick Inventory of Depressive Symptoms (QIDS-SR; Rush et al., 2003) and to meet DSM-5 criteria for Major Depressive Disorder according to the Mini International Neuropsychiatric Interview (MINI; Sheehan et al., 1997). Participants were excluded if they had current or past bipolar disorder, psychotic disorder, and/or schizophrenia.

Participants in the Control group had mean age of 23 (range 19-35) and 73\% $(\mathrm{n}=22)$ were female. Self-reported ancestry was as follows: $40 \%(\mathrm{n}=12)$ were European, $30 \%$ $(\mathrm{n}=9)$ were Asian, $17 \%(\mathrm{n}=5)$ were African-American or Black, 3\% ( $\mathrm{n}=1)$ were American Indian or Alaska Native, $13 \%(n=4)$ were more than one race or reported that none of the categories were applicable and 28\% ( $\mathrm{n}=11)$ were Hispanic. Participants were excluded if they had any current or past psychiatric disorder. They were screened to have a score of 6 or less in the QIDS and to never had experienced an episode of MDD. Participants were excluded if they had any current or past psychiatric disorder. All participants were compensated at a rate of $\$ 20 /$ hour.

\section{Materials}

QIDS-SR (Rush et al., 2003): a 16-item self-report questionnaire that assesses the nine diagnostic symptom domains used to characterize a major depressive episode. The psychometric properties of the QIDS-SR are very good and detailed in the Supplementary Material.

MINI (Sheehan et al., 1997): Research assistants trained on diagnostic interviewing completed in-person interviews for eligible participants, using version 7.2 of the Mini International Neuropsychiatric Interview for the DSM-5. The MINI is a standardized instrument used for brief screenings to diagnose a variety of psychiatric disorders. More details about interviewer training and evaluation can be found in the Supplementary Material.

\section{Stimuli}

A set of highly-controlled face stimuli was created. We developed four three-dimensional face models using the software MakeHuman v1.1.0 (http://www . makehumancommunity.org/). Identical models for eyeballs, eyebrows, skin and teeth were used across identities. MakeHuman allows users to develop expression pose models independently from the identity shape models. Thus, the exact same expression pose model was applied to all identities. We used pose models for happiness and sadness developed and validated in previous research (Hays et al., 2020). We applied these pose models to the four face identity models, producing a total of 12 combinations of expression (neutral, sad, and happy) and identity (four male identities) models. Each model was rendered to a high-resolution image, from a frontal viewpoint (see examples in Figure 1b and all models in Figure S1 of the Supplementary Material).

We used JPsychoMorph 1.0 to morph the original images and obtain intermediate levels of identity difference and emotional expression, in $10 \%$ increment steps. We then performed a pilot study to obtain psychometric curves that allowed us to choose levels of identity and emotional expression yielding a given discrimination performance (for details, see Supplementary Material).

Three stimulus sets were created based on the results of the pilot study. Identities were grouped into two stimulus sets: "Bob vs. Joe" and "Sam vs. Tom". There were two versions of the first stimulus set: non-ambiguous, with stimuli yielding approximately $d^{\prime}=2.0$ in the pilot study, and ambiguous, with stimuli yielding approximately $d^{\prime}=1.25$ in the pilot study. The second stimulus set had only a nonambiguous version (i.e., $d^{\prime}=2.0$ ).

\section{Procedure}

The study consisted of three separate sessions of about 90 minutes each, all identical except for the stimulus set presented to the participants. The first and second sessions involved the non-ambiguous "Bob vs. Joe" and "Sam vs. Tom" stimulus sets, respectively. The third session involved the ambiguous "Bob vs. Joe" stimulus set.

We included a brief familiarization procedure at the beginning of each session. Participants were instructed that their first task would be to learn the faces of two unfamiliar people. They were asked to memorize the faces and their names, and warned that later their recognition of the faces would be tested. These instructions were followed by the presentation of two $60 \mathrm{~s}$ videos, each showing a different face through changes in camera viewpoint and emotional expression (neutral, happy, angry, fearful, disgusted, sad, and surprised). Each video was repeated twice, accompanied by the name of the face presented (Bob, Joe, Sam, or Tom) and instructions to memorize the face.

After familiarization, participants were presented with three different identification tasks, separated by resting periods of 2 minutes in duration. The order of presentation of each task was shuffled for each participant and session. The three tasks differed only on the emotional expressions involved: neutral vs. sad, neutral vs. happy, and sad vs. happy. Each combination of identity and emotional expression was reported by the participants through a different key. 
One identity was assigned to the "left" keys: "Q" for happy, "A" for neutral, and "Z" for sad. The other identity was assigned to the "right" keys: "Y" for happy, "G" for neutral, and "V" for sad. Note that "top" keys were always assigned to happy, "middle" keys to neutral, and "bottom" keys to sad. Only four of the keys were functional (i.e., recorded a response and advanced the trial) in each task.

At the beginning of each task, instructions were displayed indicating that the participant's task would be to identify four faces, each assigned to a single response key. The four faces were shown, labeled with each face's name, emotional expression, and response key. The instructions also highlighted that the left keys were assigned to one identity and the right keys to the other, and that the top keys were assigned to one expression and the bottom keys to the other. Participants were warned that faces would be shown very briefly and were instructed to respond as accurately and as fast as possible.

Each task consisted of 20 blocks of 20 trials each, 400 trials total. Each block involved 5 presentations of each of the four stimuli and trials were randomized within blocks. A trial started with the presentation of a white fixation cross-hair in the middle of the screen for $500 \mathrm{~ms}$, followed by the presentation of the face stimulus for $200 \mathrm{~ms}$. The trial ended either with the participant's response or when $2 \mathrm{~s}$ passed since the presentation of the face stimulus, whichever happened first. Participants were given feedback about the correctness of their responses, consisting of the word "Correct!" in blue, or the word "Incorrect!" in red. When participants failed to respond before the deadline, they saw the words "Too Slow!" in red. There was a $1 \mathrm{~s}$ inter-trial interval before the start of the next trial.

\section{Model-Based Data Analysis}

The data from each task within a session were analyzed separately.

SDT analyses assume that performance is the result of a well-learned task. Thus, we discarded data from the early period during which participants were still learning the task. Learning curves were obtained by averaging performance within a moving window of 100 trials, starting with trials 1-100, then trials 2-101, and so on. An exponential function was fitted to such learning curves, and the trial in which the slope of the fitted curve was smaller than .001 for the first time was the chosen as a cutoff: only subsequent trials were included in the final analysis. In addition, SDT analyses require that performance be well-above chance and below perfect, so data from participants who had a performance below $40 \%$ correct or above $95 \%$ were excluded. Detailed information about the number of participants included in each analysis and their accuracy in the identification task is reported in Table S1 of the Supplementary Material.

Data from each group and each identification task were separately fitted to GRT with individual differences (GRT-
wIND; Soto et al., 2015), using procedures detailed in the Supplementary Material and implemented in the $\mathrm{R}$ package grtools (Soto et al., 2017). Once the best-fitting parameters were obtained, they were used to compute measures of discriminability and separability for each face dimension. Discriminability was measured by computing $d^{\prime}$ directly from the model for each participant (see Figure 1a). The mean discriminability is reported. Threshold bias was measured by computing distance of the bound from the center point across perceptual distributions. Deviations of perceptual separability were measured by computing the $L 1$ distance between distributions (shaded area in Figure 1b), and deviations of decisional separability by computing the degree of rotation of bounds (shaded angular distance in Figure 1b).

We used a parametric bootstrap procedure (Good, 2006) to obtain $95 \%$ confidence intervals on these measures. Each step in the procedure consists of generating a new data sample from the fitted model, and then fitting the GRT-wIND model to such simulated data. The obtained parameter values can then be used to compute the measures described in the previous paragraph. This process was repeated 1,000 times for each model, resulting on an empirical distribution of the measures, which was used to directly obtain $95 \%$ confidence intervals using the simple percentile method (i.e., 25th and 975th 1,000-quantiles). We used a parametric rather than a non-parametric bootstrap procedure because (1) our analysis assumes a model for the data distribution, so in this case the parametric bootstrap is appropriate, and (2) using the nonparametric bootstrap with small sample sizes $(<100)$ such as ours leads to imprecise confidence intervals (Good, 2006). We considered cases in which the confidence intervals for the Control and MDD groups did not overlap as suggesting a reliable difference between the groups for that specific comparison. This is considered a rather conservative method to detect differences between groups (Schenker and Gentleman, 2001), corresponding to a test with $\alpha \leq .01$ (Cumming and Finch, 2005).

\section{Results}

The mean QIDS depression score was significantly higher for MDD $(m=14.0, s . d .=3.86)$ than for control $(m=2.13$, $s . d .=1.72)$ participants, $t(40.04)=15.39, p<.001$. On the other hand, groups did not differ in mean percentage of participants excluded in total (MDD: $m=9.6$, s.d. $=5.4$; Control: $m=14.1$, s.d. $=6.4 ; t(15.54)=1.59, p>.1)$, excluded due low performance (MDD: $m=4.8$, s.d. $=3.8$; Control: $m=8.5$, $s . d .=6.3 ; t(13.12)=1.52, p>.1)$, excluded due to high performance (MDD: $m=4.4$, s.d.=3.3; Control: $m=5.2$, s.d. $=2.9$; $t(15.75)=0.5, p>.1)$, or in percentage of trials excluded from the data of included participants (MDD: $m=3.4$, s.d. $=10.2$; Control: $m=3.5$, s.d. $=9.1 ; t(471.86)=0.12, p>.1)$. The Supplementary Material includes plots of average confusion matrices (Figures S3-S4; we do not discuss such matrices here 
because the patterns of results captured by the model parameters are not easily discernible from the raw data) and detailed information about the fit of the model to data (Table S2). The model showed excellent fit to the data, with all $R^{2}$ values in the $.98-.99$ range.

The Supplementary Material also includes simulations showing that parameters of the model show very good recoverability and identifiability with the amount of data gathered here, and that the model can effectively dissociate between perceptual and decisional factors contributing to the confusion data.

The main results are shown in Table 1, which is subdivided into six analyses described below. In each case, the table includes estimates of the statistic used and its $95 \%$ confidence interval for each group. Highlighted in gray shading are all the cases in which the confidence intervals for the Control and MDD groups did not overlap. The same results are shown in a graphical form in Figures S1-S6 of the Supplementary Material.

\section{Perceptual Effects}

Perceptual Discriminability of Emotion. Table 1a shows the results of our analysis of emotion discriminability in terms of $d^{\prime}$ (the distance between perceptual distributions shown in Figure 1a). For the non-ambiguous stimuli used in the first two sessions, an impairment in discriminability was observed for the MDD group only in the Neutral vs. Happy task. The same pattern of results was observed with the two stimulus sets, and thus this seems like a reliable effect that generalizes across identities. Thus, people with MDD appear to have more difficulty discriminating neutral versus happy emotion with unambiguous stimuli.

With increased ambiguity during the third session, an impairment in discriminability of face emotion was observed across all emotion pairs (bottom panel) for the MDD group compared to the control group. This general deficit with ambiguous stimuli is in line with previous reports (Schepman et al., 2012).

Perceptual Separability of Emotion - Table 1b shows the results of our analysis of perceptual separability of emotion-that is, whether the perception of emotion is influenced by face identity. The wealth of the evidence suggests that MDD does not influence the perceptual separability of emotion. The $L 1$ distances between recovered perceptual distributions (shaded areas in Figure 1b) were very similar between groups for most tasks and stimulus sets, and the 95\% confidence intervals overlapped in most cases. Increasing face ambiguity did not change the results, contrary to what was found in the analysis of emotion discriminability reported above.

The only exception was a decrease in perceptual separability (i.e., increase in $L 1$ distance) for the MDD group in the Neutral vs. Sad discrimination, but only for the second pair of identities. This suggests that some specific identities might influence the detection of sadness, perhaps because shape features that are specific to some identities are confused with features of sadness. However, we see very little value in drawing conclusions from such context-dependent results. In sum, the overall pattern of results suggests that depression does not influence the perceptual separability of emotion.

Perceptual Separability of Identity . Table 1c shows the results of our analysis of perceptual separability of identity-that is, whether the perception of identity is influenced by face emotion. Here, all of the evidence suggests that MDD does not influence the perceptual separability of identity. The recovered perceptual distributions and their $L 1$ distances are very similar between groups for all tasks and stimulus sets, and the $95 \%$ confidence intervals on the $L 1$ distances overlapped in all cases. The result generalizes across identities and is not influenced by face ambiguity.

This result is at odds with previously-reported impairments in filtering of information about emotional expression when identity is being classified (Gilboa-Schechtman et al., 2004). Because the selective attention task used by GilboaSchechtman and colleagues cannot distinguish between perceptual and decision-making mechanisms of interaction between dimensions, the most likely explanation for their results is that the observed impairment was not perceptual in nature, but stems from decision-making mechanisms.

Summary of Perceptual Effects. We only found consistent perceptual effects of depression on the perceptual discriminability of emotion. In particular, happiness was in general more difficult to detect by MDD patients, and there was a general deficit on emotion discrimination with ambiguous stimuli. The results suggest that there are no effects of depression on perceptual separability of emotion or identity. In sum, the results suggest that depression does not influence the perceptual separability of identity.

\section{Decisional Effects}

Threshold Bias of Emotion. Table 1d shows the results of our analysis of threshold bias (the distance of the bound from the center of the model shown in Figure 1a) in the discrimination of emotion. Here, all of the evidence suggests that MDD does not influence decision bias, as the $95 \%$ confidence intervals on threshold bias overlapped in all cases. The result generalizes across identities and is not influenced by face ambiguity.

Decisional Separability of Emotion. Table 1e shows the results of our analysis of decisional separability of emotion-that is, whether decisions about emotion were influenced by face identity. Here, deviations from decisional separability are measured through mean degree of rotation (the "angular distance" shown in Figure 1b). The results suggest that it is possible to find group differences in decisional sep- 


\begin{tabular}{|c|c|c|c|c|c|c|c|}
\hline \multirow[t]{3}{*}{ Analysis } & \multicolumn{6}{|c|}{$\begin{array}{c}\text { Statistics } \\
\text { (95\% confidence interval within brackets) }\end{array}$} & \multirow[t]{3}{*}{ Conclusion } \\
\hline & \multicolumn{2}{|c|}{ Neutral vs. Sad } & \multicolumn{2}{|c|}{ Neutral vs. Happy } & \multicolumn{2}{|c|}{ Happy vs. Sad } & \\
\hline & Control & MDD & Control & MDD & Control & MDD & \\
\hline \multirow{2}{*}{\multicolumn{8}{|c|}{$\begin{array}{l}\text { (a) Perceptual Discriminability of } \\
\text { Emotion }\end{array}$}} \\
\hline & \\
\hline Does MDD influence it? & $\begin{array}{c}2.83 \\
{[2.79,2.99]}\end{array}$ & $\begin{array}{c}2.76 \\
{[2.72,2.91]}\end{array}$ & $\begin{array}{c}3.40 \\
{[3.35,3.59]}\end{array}$ & $\begin{array}{c}3.17 \\
{[3.13,3.33]}\end{array}$ & $\begin{array}{c}3.95 \\
{[3.94,4.23]}\end{array}$ & $\left.\begin{array}{c}3.86 \\
{[3.85,4.13]}\end{array}\right]$ & $\begin{array}{l}\text { Only for happiness. Discriminability } \\
\text { of happiness was impaired in MDD }\end{array}$ \\
\hline $\begin{array}{l}\text { Does the result depend on specific face } \\
\text { identities? }\end{array}$ & $\begin{array}{c}2.56 \\
{[2.51,2.69]}\end{array}$ & $\begin{array}{c}2.54 \\
{[2.51,2.67]}\end{array}$ & $\begin{array}{c}3.74 \\
{[3.72,3.96]}\end{array}$ & $\begin{array}{c}3.32 \\
{[3.30,3.52]}\end{array}$ & $\begin{array}{c}3.99 \\
{[3.97,4.29]}\end{array}$ & $\begin{array}{c}3.84 \\
{[3.85,4.11]}\end{array}$ & $\begin{array}{l}\text { No. } \\
\text { Results remained the same when identities } \\
\text { were changed. }\end{array}$ \\
\hline $\begin{array}{l}\text { Does the result depend on face } \\
\text { information ambiguity? }\end{array}$ & $\begin{array}{c}2.70 \\
{[2.65,2.84]}\end{array}$ & $\begin{array}{c}2.46 \\
{[2.42,2.60]}\end{array}$ & $\begin{array}{c}3.39 \\
{[3.37,3.61]}\end{array}$ & $\begin{array}{c}2.99 \\
{[2.97,3.15]}\end{array}$ & $\begin{array}{l}3.76 \\
{[3.76,4.02]}\end{array}$ & $\begin{array}{c}3.51 \\
{[3.43,3.73]}\end{array}$ & $\begin{array}{l}\text { Yes. } \\
\text { Ambliguty produced porer discriminability } \\
\text { for MDD across all emotion pairs. }\end{array}$ \\
\hline
\end{tabular}

(b) Perceptual Separability of

Emotion

Statistic used: $L l$ distance

\begin{tabular}{|c|c|c|c|c|c|c|c|}
\hline Does MDD influence it? & $\begin{array}{c}.067 \\
{[.057, .081]}\end{array}$ & $\begin{array}{c}.067 \\
{[.053, .084]}\end{array}$ & $\begin{array}{c}.067 \\
{[.051, .085]}\end{array}$ & $\begin{array}{c}.040 \\
{[.024, .057]}\end{array}$ & $\begin{array}{c}.014 \\
{[.003, .030]}\end{array}$ & $\begin{array}{c}.018 \\
{[.008, .038]}\end{array}$ & 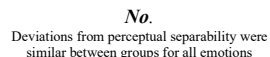 \\
\hline $\begin{array}{l}\text { Does the result depend on specific face } \\
\text { identities? }\end{array}$ & $\begin{array}{c}.028 \\
{[.013, .047]}\end{array}$ & $\begin{array}{c}.064 \\
{[.052, .082]}\end{array}$ & $\begin{array}{l}.004 \\
{[.002, .026]}\end{array}$ & $\begin{array}{c}.019 \\
{[.006, .036]}\end{array}$ & $\begin{array}{c}.013 \\
{[.003, .032]}\end{array}$ & $\begin{array}{c}.013 \\
{[.006, .035]}\end{array}$ & $\begin{array}{l}\text { Only for sadness. } \\
\text { viations from perceptual sep } \\
\text { being stronger for } M D D\end{array}$ \\
\hline $\begin{array}{l}\text { Does the result depend on face } \\
\text { information ambiguity? }\end{array}$ & $\begin{array}{l}.036 \\
{[.026, .050]}\end{array}$ & $\begin{array}{c}.040 \\
{[.031, .059]}\end{array}$ & $\begin{array}{c}.065 \\
{[.049, .084]}\end{array}$ & $\begin{array}{c}.049 \\
{[.034, .063]}\end{array}$ & $\begin{array}{c}.015 \\
{[.006, .032]}\end{array}$ & $\begin{array}{c}.019 \\
{[.010, .036]}\end{array}$ & $\begin{array}{l}\text { No. } \\
\text { Results did not change when ambigutity was } \\
\text { inicreased }\end{array}$ \\
\hline
\end{tabular}

(c) Perceptual Separability of Identity

Statistic used: $L l$ distance

Does MDD influence it? Does the result depend on specific face
identities?

Does the result depend on face information ambiguity?

\begin{tabular}{|c|c|c|c|c|c|c|}
\hline $\begin{array}{c}.034 \\
{[.025, .053]}\end{array}$ & $\begin{array}{c}.040 \\
{[.030, .054]}\end{array}$ & $\begin{array}{c}.098 \\
{[.085, .111]}\end{array}$ & $\begin{array}{c}.096 \\
{[.084, .108]}\end{array}$ & $\begin{array}{c}.045 \\
{[.031, .059]}\end{array}$ & $\begin{array}{c}.066 \\
{[.050, .083]}\end{array}$ & $\begin{array}{l}\text { No. } \\
\text { Deviations from perceptual separability were } \\
\text { similar between groups for all emotions. }\end{array}$ \\
\hline $\begin{array}{c}.070 \\
{[.055, .088]}\end{array}$ & $\begin{array}{c}.039 \\
{[.028, .058]}\end{array}$ & $\begin{array}{c}.099 \\
{[.087, .114]}\end{array}$ & $\begin{array}{c}.098 \\
{[.084, .118]}\end{array}$ & $\begin{array}{c}.101 \\
{[.082, .117]}\end{array}$ & $\begin{array}{c}.109 \\
{[.097, .127]}\end{array}$ & $\begin{array}{l}\text { No. } \\
\text { Results did not change when the two } \\
\text { identities presented were changed. }\end{array}$ \\
\hline $\begin{array}{c}.051 \\
{[.035, .068]}\end{array}$ & $\begin{array}{c}.041 \\
{[.028, .060]}\end{array}$ & $\begin{array}{c}.007 \\
{[.002, .025]}\end{array}$ & $\begin{array}{c}.015 \\
{[.004, .030]}\end{array}$ & $\begin{array}{c}.034 \\
{[.027, .053]}\end{array}$ & $\begin{array}{c}.020 \\
{[.009, .036]}\end{array}$ & $\begin{array}{l}\text { No. } \\
\text { Results did not thange when ambiguity was } \\
\text { increased. }\end{array}$ \\
\hline
\end{tabular}

(d) Threshold Bias of Emotion

Statistic used: Median distance from center

\begin{tabular}{|c|c|c|c|c|c|c|c|}
\hline Does MDD influence it? & $\begin{array}{c}.02 \\
{[-.08, .09]}\end{array}$ & {$\left[\begin{array}{c}-.01 \\
{[-.07, .05]}\end{array}\right.$} & $\begin{array}{c}-.04 \\
{[-.12, .02]}\end{array}$ & {$\left[\begin{array}{c}-.10 \\
{[-.17,-.03]}\end{array}\right.$} & $\begin{array}{c}-.05 \\
{[-.11, .02]}\end{array}$ & {$[-.07,-.14]$} & $\begin{array}{l}\text { No. } \\
\begin{array}{l}\text { No differences in threshold bias were found } \\
\text { between groups. }\end{array}\end{array}$ \\
\hline $\begin{array}{l}\text { Does the result depend on specific face } \\
\text { identities? }\end{array}$ & $\begin{array}{c}-.04 \\
{[-.13, .00]}\end{array}$ & $\begin{array}{c}.02 \\
{[-.08, .05]}\end{array}$ & $\begin{array}{c}-.10 \\
{[-.19,-.04]}\end{array}$ & $\begin{array}{c}-.09 \\
{[-.16,-.03]}\end{array}$ & $\begin{array}{c}-.09 \\
{[-.19,-.03]}\end{array}$ & $\begin{array}{c}-.12 \\
{[-.22,-.07]}\end{array}$ & $\begin{array}{l}\text { No. } \\
\text { Results did not change when the two } \\
\text { identities presented were changed.. }\end{array}$ \\
\hline $\begin{array}{l}\text { Does the result depend on face } \\
\text { information ambiguity? }\end{array}$ & {$\left[\begin{array}{c}-.11 \\
{[-.18,-.058]}\end{array}\right.$} & $\begin{array}{c}-.02 \\
{[-.061, .09]}\end{array}$ & $\begin{array}{c}-.07 \\
{[-14, .00]}\end{array}$ & $\begin{array}{c}-.02 \\
{[-.08, .06]}\end{array}$ & $\begin{array}{c}-.09 \\
{[-.18,-.03]}\end{array}$ & $\begin{array}{c}-.08 \\
{[-.16,-.03]}\end{array}$ & $\begin{array}{l}\text { No. } \\
\text { Results did not change when ambiguity was } \\
\text { increased. }\end{array}$ \\
\hline
\end{tabular}

(e) Decisional Separability of

Emotion

Statistic used: Mean degree of rotation

\begin{tabular}{|c|c|c|c|c|c|c|c|}
\hline Does MDD influence it? & $\begin{array}{c}5.8 \\
{[3.4 .8 .8]}\end{array}$ & $\begin{array}{c}1.5 \\
{[-0.8,4.2]}\end{array}$ & $\begin{array}{c}11.3 \\
{[9.7,13.7]}\end{array}$ & $\begin{array}{c}6.3 \\
{[4.6,8.6]}\end{array}$ & $\begin{array}{c}5.9 \\
{[4.6,7.9]}\end{array}$ & $\begin{array}{c}9.9 \\
{[8.0,12.9]}\end{array}$ & $\begin{array}{l}\text { Yes. } \\
\text { Deviations from decisional separability } \\
\text { differed between groups for some emotions. }\end{array}$ \\
\hline $\begin{array}{l}\text { Does the result depend on specific face } \\
\text { identities? }\end{array}$ & $\begin{array}{c}3.2 \\
{[0.9,5.4]}\end{array}$ & $\begin{array}{c}-3.8 \\
{[-6.1,-1.5]}\end{array}$ & $\begin{array}{c}4.5 \\
{[2.4,6.7]}\end{array}$ & $\begin{array}{c}6.6 \\
{[3.6,9.5]}\end{array}$ & $\begin{array}{c}4.6 \\
{[2.5,6.9]}\end{array}$ & $\begin{array}{c}3.6 \\
{[1.6,6.2]}\end{array}$ & $\begin{array}{l}\text { Yes. } \\
\text { Differences were found again, but the pattern } \\
\text { changed with change in identity. }\end{array}$ \\
\hline $\begin{array}{l}\text { Does the result depend on face } \\
\text { information ambiguity? }\end{array}$ & $\begin{array}{c}7.4 \\
{[5.4,10.0]}\end{array}$ & $\begin{array}{c}9.0 \\
{[6.3,11.6]}\end{array}$ & $\begin{array}{c}9.1 \\
{[6.1,12.2]}\end{array}$ & $\begin{array}{c}6.1 \\
{[3.5,8.5]}\end{array}$ & $\begin{array}{c}6.4 \\
{[4.2 .9 .4]}\end{array}$ & $\begin{array}{c}9.5 \\
{[7.6,11.8]}\end{array}$ & $\begin{array}{l}\text { Yes. } \\
\text { No differences between groups were found } \\
\text { when ambiguity was increased. }\end{array}$ \\
\hline
\end{tabular}

(f) Decisional Separability of Identity

Statistic used: Mean degree of rotation

Does MDD influence it?

Does the result depend on specific face identities?

Does the result depend on face information ambiguity?

\begin{tabular}{|cccc}
\hline $\mathbf{0 . 0}$ & $\mathbf{- 1 0 . 4}$ & -3.7 & -2.5 \\
\hline $\mathbf{- 2 . 7}, \mathbf{1 . 8}]$ & {$[-\mathbf{1 2 . 2}, \mathbf{- 8 . 2}]$} & {$[-5.3,-2.5]$} & {$[-3.7,-1.3]$} \\
\hline 0.8 & -3.1 & 0.3 & -1.6 \\
{$[-1.3,2.6]$} & {$[-5.4,-0.1]$} & {$[-1.2,1.6]$} & {$[-3.9,0.4]$} \\
\hline $\mathbf{- 6 . 9}$ & $\mathbf{- 1 . 3}$ & 8.7 & 5.4 \\
\hline$-\mathbf{8 . 5}, \mathbf{- 5 . 4}]$ & {$[-\mathbf{3 . 8}, \mathbf{0 . 7}]$} & {$[6.0,10.6]$} & {$[3.7,7.2]$}
\end{tabular}

\begin{tabular}{|c|c|c|}
\hline $\begin{array}{c}1.2 \\
{[0.1,2.0]}\end{array}$ & $\begin{array}{c}--2.6 \\
{[-4.7,-1.2]}\end{array}$ & $\begin{array}{l}\text { Yes. } \\
\text { Deviations from decisional separability } \\
\text { differed between groups for some emotions }\end{array}$ \\
\hline $\begin{array}{c}-4.8 \\
{[-6.6,-2.8]}\end{array}$ & $\begin{array}{c}-6.9 \\
{[-8.8,-5.4]}\end{array}$ & $\begin{array}{l}\text { Yes. } \\
\text { Differences between groups were not found } \\
\text { when the identities were changed.. }\end{array}$ \\
\hline $\begin{array}{c}3.5 \\
{[1.7,4.9]}\end{array}$ & $\begin{array}{c}3.9 \\
{[2.3,5.5]}\end{array}$ & $\begin{array}{l}\text { Yes. } \\
\text { The pattern of differences changed when } \\
\text { ambiguity was increased. }\end{array}$ \\
\hline
\end{tabular}

Table 1

Results of the model-based analysis of data using signal detection theory. For each case, estimates of the statistic used and their 95\% confidence intervals are provided for each group. Highlighted in gray shading are all the cases in which the confidence intervals for the Control and MDD groups did not overlap, suggesting a reliable difference between groups. 
arability, but they are highly inconsistent. For example, in the first session we find that the MDD group shows more separability (i.e., lower degree of rotation) than the Control group for Neutral vs. Happy, but less separability for Happy vs. Sad. When identities were changed, both effects disappeared and instead we found a significant difference for Neutral vs. Sad. Similarly, all effects disappeared when participants were presented with ambiguous faces. The results suggest that MDD patients use different decisional strategies from controls, but that they depend strongly on the presented stimulus set.

Decisional Separability of Identity. Table $1 \mathrm{f}$ shows the results of our analysis of decisional separability of identity-that is, whether decisions about identity were influenced by face emotion. As before, it is possible to find group differences, but results are inconsistent across analyses. Significant differences were found for Neutral vs. Sad, both with the original stimulus set and its ambiguous version, but these effects are in opposite directions. A significant difference was also found for Happy vs. Sad with the original stimulus set. As before, we interpret these results as suggesting that MDD patients use different decisional strategies from controls, but that they depend strongly on the presented stimulus set.

Summary of Decisional Effects. Our results suggest that depression does not influence overall threshold biases in the discrimination of emotion in our task. However, we found that MDD patients do use different decisional strategies than those shown by control participants, but that those differences show absolutely no consistency across stimulus sets.

\section{Discussion}

In this study, we used a highly controlled stimulus set and an SDT model-based analysis to determine whether any perceptual biases exist in the recognition of face emotion in depression, aside from higher-level decisional and cognitive biases. We found that MDD patients were in general impaired in their ability to detect happiness (i.e., discriminate it from neutral faces), regardless of face identity and ambiguity. This is in line with the prediction of cognitive theories of major depression (Beck, 2008; Disner et al., 2011) and with several previous reports in the literature (reviewed in Bistricky et al., 2011). Inaccurate recognition of happy expressions in MDD has not been consistently established in the prior literature (Bourke et al., 2010), but this might partly be due to a lack of control of stimulus factors (e.g., intensity of expressions) and decisional biases.

We also found that face ambiguity has an important role on whether or not perceptual effects can be found, in accordance to previous studies (Schepman et al., 2012). Impairments in discriminability of all pairs of emotional expressions were found when ambiguous stimuli were used. The modulation of perceptual deficits by expression inten- sity might be one of the reasons why some prior studies have found global deficits of facial emotion processing or no deficits at all (for a review, see Bourke et al., 2010), with the other likely culprits being lack of control for expression intensity and decisional biases.

There was little evidence suggesting that MDD influences perceptual interactions between face expression and identity. Thus, there are no reasons to expect that people with depression would have trouble "filtering out" information about emotional expression when they perceptually process face identity, as previously suggested (Gilboa-Schechtman et al., 2004). A likely possibility is that prior results stem from decisional rather than perceptual mechanisms. We found that MDD patients show different patterns of violation of decisional separability than control participants, but that those differences are not consistent across stimulus sets. Determining exactly what factors can explain differences in decisional strategies will require further research.

Our results also suggest that people with depression should have no trouble "filtering out" information about identity when they perceptually process face expression. This is important because generalization of face expression learning to unseen identities might depend on such ability to "filter out" identity information (Soto and Ashby, 2019). Such feedback-based expression learning may include ABM, but not be limited to it.

In sum, the most important and consistent results we found were perceptual in nature and focused on the discriminability of face emotion, with MDD patients showing lower sensitivity to detect happiness, and to discriminate emotion in ambiguous faces.

Interpersonal theories suggest that people with depression are alert for signs of negative social feedback (Joiner and Metalsky, 1995; Surguladze et al., 2004). On the contrary, in our results depression was associated mainly with a reduction in responsiveness to happy faces. If our results generalize from our highly controlled tasks to the real world, we would expect depression to be accompanied by a dulled sensitivity to positive social feedback, either alone or in addition to the heightened sensitivity to negative social feedback proposed by interpersonal theories.

The results of prior studies on face perception biases in depression have been highly heterogeneous (for reviews, see Bistricky et al., 2011; Bourke et al., 2010). Such heterogeneity could result from a lack of control for emotion intensity in the stimuli and from decisional factors, both of which showed to be important in our study. In addition, anxiety often co-occurs with depression (Kessler and Walters, 1998), and it might produce an independent effect on expression perception. An important next step for computational work in this area would be to use computational modeling to understand idiosyncratic ways in which expression processing is affected in specific individuals and its relation to symptoma- 
tology.

Our stimuli and design also have some limitations compared to other studies. For example, our methods require many trials with the same faces, which restricts the number of identities and emotions that our can be tested. We focused on male, Caucasian faces, and a single pose model for each expression. This allowed tight stimulus control, but more research is necessary to test the generalizability of our results. A final important point is that while current feedbackbased treatments attempt to reduce biases towards negative face expressions, the results reported here suggest that more attention should be paid at increasing perceptual sensitivity to evidence of positive face expressions (e.g., happiness) as a target for treatments.

\section{Mechanisms of Face Perception Bias in Depression: A Working Hypothesis}

An advantage of SDT is that it can be linked to channel models from the psychophysics literature (e.g., Series et al., 2009; Soto et al., 2018), which allow one to propose mechanistic explanations for differences in perceptual discriminability. We finish this work by taking advantage of this link, and propose a computational model of face perception biases in depression. A large body of work supports a twochannel opponent coding system for face emotion (e.g., Burton et al., 2015; Cook et al., 2011), illustrated in the left part of Figure 2a. In this model, level of happiness is encoded by two channels, represented in the figure by curves of different color. The red channel responds most strongly to happy expressions, and its response decreases with less evidence of happiness in a face. The black channel responds most strongly to an anti-happy expression, an expression with the opposite features as happiness, in relation to a neutral expression. When a face is presented to this model, it outputs two channel responses, which include channel noise added during processing. Note that the happiness level is not explicitly represented in the channel responses; perceptual evidence for happiness must be computed from the channels' output. Because of channel noise, the computed perceptual evidence is also noisy (i.e., the purple distribution in Figure 2a), just as assumed by SDT's representation of happiness (see a full formal description in the Supplementary Material).

We assume that in depression the channels encoding happiness and other rewarding emotions show suppressed activity, as shown by the orange arrow in Figure 2b. Such channels are correlated with the representation of anti-sadness (Hsu and Young, 2004; Rutherford et al., 2008), so we simplify the model by having a single continuum going from sadness to happiness.

Figure 2c shows a simulation of the discriminability of happy and sad expressions against neutral, as a function of expression intensity. More ambiguous expressions are closer to zero at the middle of the scale. The simulation repro-

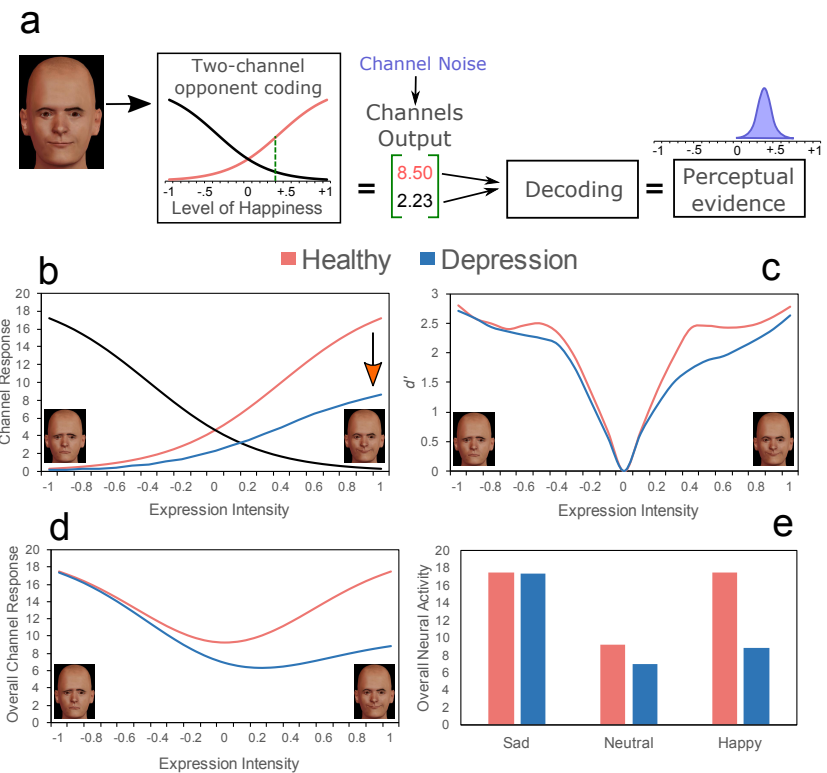

Figure 2. A model of face expression encoding in depression. The main model in (a) is composed of a two-pool opponent coding system for face expression that has empirical support in the previous literature, which together with optimal decoding produces the distributions of perceptual evidence for a given expression assumed by signal detection theory. The main assumption of the model in (b) is that in depression the neural populations encoding positive expressions show suppressed activity. This assumption is enough to explain (c) the drop in sensitivity $\left(d^{\prime}\right)$ found for happy expressions regardless of intensity and for low-intensity (i.e., highly ambiguous) sad expressions found in our study, and (d-e) the drop in the ability of relatively positive expressions to compete for attention when presented with relatively negative expressions, as well as the attenuated neural activity elicited by positive expressions.

duces the asymmetrical results found in the present study: in depression (blue curve) there is a large drop in sensitivity to happy expressions throughout most values of intensity, whereas the same drop is found for sad faces only at lower levels of intensity (i.e., high ambiguity). That is, the model explains deficits in the detection of sadness in ambiguous stimuli as an indirect result of suppressed representation of positive expressions, in an opponent-channel system.

The model also predicts prior results that it was not explicitly designed to explain, like evidence of unimpaired and unbiased identification of unambiguous expressions (see Bistricky et al., 2011). In Figure 2c, as expression intensity increases, the gap between blue and red curves becomes smaller and eventually closes for happy expressions, as shown for sad expressions.

A number of studies have used paradigms in which multi- 
ple faces are displayed simultaneously to determine whether people with depression show biased attention towards negative or away from positive expressions. Figure $2 d$ shows the overall channel activity (i.e., the sum of activity in the two channels) produced by a variety of expressions in a negativepositive continuum, and Figure $2 \mathrm{e}$ shows the specific values expected for unambiguously sad, neutral, and happy faces. Assuming that attention is biased toward a face proportionally to this overall activity, differences in height between bars represent the relative ability of the stimuli to compete for attention if they were presented in a display together. The model predicts that depression would be accompanied by a drop in the ability of happy faces to compete for attention, either against neutral or sad faces, as well as a smaller increase in the ability of sad faces to compete for attention against neutral faces. Several studies have confirmed such predictions (for a review, see Bistricky et al., 2011), although inconsistently.

We must stress that the simulations in Figure 2 are presented as a proof of concept rather than as a full-fledged model of face perception biases in depression. Such a theory would require additional details and is outside the scope of this study. However, our simulations show that it is possible to formalize mechanistic explanations of perceptual biases in depression that make clear and quantitative predictions.

One result that is not explained by this model is that people with depression consistently show a tendency to interpret ambiguous/neutral stimuli as negative (for a review, see Bourke et al., 2010). Here, we found no evidence that such an effect stems from a decisional bias. Assuming that the effect is truly perceptual, a more complete version of the model might explain such results by sub-optimal computation of evidence for emotion (Series et al., 2009).

\section{Conclusion}

The present work is a step forward toward a better understanding of the mechanisms underlying face perception biases in depression, and toward formalizing the assumptions behind cognitive theories of depression in the form of concrete, testable computational models. Face perception is a particularly advantageous area for such developments, as faces are complex stimuli with social significance, but they are also easy to manipulate and intensely studied in basic research. We hope that the approach taken here can be expanded to incorporate other biases found in depression.

Acknowledgments. Research reported here was supported by the National Institute of Mental Health under Award Number R21MH112013 to Fabian A. Soto and Christopher G. Beevers. The content is solely the responsibility of the authors and does not necessarily represent the official views of the National Institutes of Health. S.D.G.

\section{References}

Abela, J. R. Z. and Hankin, B. L. (2011). Rumination as a vulnerability factor to depression during the transition from early to middle adolescence: A multiwave longitudinal study. Journal of Abnormal Psychology, 120(2):259-271.

Ashby, F. G. and Soto, F. A. (2015). Multidimensional signal detection theory. In Busemeyer, J., Townsend, J. T., Wang, Z. J., and Eidels, A., editors, Oxford Handbook of Computational and Mathematical Psychology, pages 13-34. Oxford University Press, New York, NY.

Ashby, F. G., Waldron, E. M., Lee, W. W., and Berkman, A. (2001). Suboptimality in human categorization and identification. Journal of Experimental Psychology: General, 130(1):77.

Bar-Haim, Y. (2010). Research review: Attention bias modification (ABM): a novel treatment for anxiety disorders. Journal of Child Psychology and Psychiatry, 51(8):859-870.

Bar-Haim, Y., Holoshitz, Y., Eldar, S., Frenkel, T. I., Muller, D., Charney, D. S., Pine, D. S., Fox, N. A., and Wald, I. (2010). Life-threatening danger and suppression of attention bias to threat. American Journal of Psychiatry, 167(6):694-698.

Bar-Haim, Y., Lamy, D., Pergamin, L., BakermansKranenburg, M. J., and Van Ijzendoorn, M. H. (2007). Threat-related attentional bias in anxious and nonanxious individuals: a meta-analytic study. Psychological Bulletin, 133(1):1-24.

Beck, A. T. (2008). The evolution of the cognitive model of depression and Its neurobiological correlates. American Journal of Psychiatry, 165(8):969-977.

Beevers, C. G., Lee, H. J., Wells, T. T., Ellis, A. J., and Telch, M. J. (2011). Association of predeployment gaze bias for emotion stimuli with later symptoms of PTSD and depression in soldiers deployed in Iraq. American Journal of Psychiatry, 168(7):735-741.

Bistricky, S. L., Ingram, R. E., and Atchley, R. A. (2011). Facial affect processing and depression susceptibility: Cognitive biases and cognitive neuroscience. Psychological Bulletin, 137(6):998-1028.

Bourke, C., Douglas, K., and Porter, R. (2010). Processing of facial emotion expression in major depression: a review. Australian and New Zealand Journal of Psychiatry, 44(8):681-696.

Burton, N., Jeffery, L., Calder, A. J., and Rhodes, G. (2015). How is facial expression coded? Journal of Vision, 15(1):1-1.

Cook, R., Matei, M., and Johnston, A. (2011). Exploring expression space: Adaptation to orthogonal and antiexpressions. Journal of Vision, 11(4):1-9.

Cumming, G. and Finch, s. (2005). Inference by eye: confidence intervals and how to read pictures of data. Amer- 
ican Psychologist, 60(2):170-180.

Disner, S. G., Beevers, C. G., Haigh, E. A. P., and Beck, A. T. (2011). Neural mechanisms of the cognitive model of depression. Nature Reviews Neuroscience, 12(8):467477.

Garner, W. R. (1974). The processing of information and structure. Lawrence Erlbaum Associates, New York.

Gilboa-Schechtman, E., Ben-Artzi, E., Jeczemien, P., Marom, S., and Hermesh, H. (2004). Depression impairs the ability to ignore the emotional aspects of facial expressions: Evidence from the Garner task. Cognition and Emotion, 18(2):209-231.

Goldstone, R. L. (1994). Influences of categorization on perceptual discrimination. Journal of Experimental Psychology: General, 123(2):178-200.

Good, P. I. (2006). Resampling methods: a practical guide to data analysis. Birkhäuser, Boston, MA.

Gotlib, I. H. and Joormann, J. (2010). Cognition and depression: Current status and future directions. Annual Review of Clinical Psychology, 6:285-312.

Green, D. M. and Swets, J. A. (1966). Signal detection theory and psychophysics. Wiley, New York.

Hays, J. S., Wong, C., and Soto, F. A. (2020). FaReT: A free and open-source toolkit of three-dimensional models and software to study face perception. Behavior Research Methods, 52(6):2604-2622.

Hsu, S. M. and Young, A. (2004). Adaptation effects in facial expression recognition. Visual Cognition, 11(7):871899.

Joiner, T. E. and Metalsky, G. I. (1995). A prospective test of an integrative interpersonal theory of depression: A naturalistic study of college roommates. Journal of Personality and Social Psychology, 69(4):778-788.

Kessler, R. C. and Walters, E. E. (1998). Epidemiology of DSM-III-R major depression and minor depression among adolescents and young adults in the national comorbidity survey. Depression and Anxiety, 7(1):314.

Leppänen, J. M. and Hietanen, J. K. (2001). Emotion recognition and social adjustment in school-aged girls and boys. Scandinavian Journal of Psychology, 42(5):429-435.

MacLeod, C. (2012). Cognitive bias modification procedures in the management of mental disorders. Current Opinion in Psychiatry, 25(2):114-120.

Marsh, A. A., Kozak, M. N., and Ambady, N. (2007). Accurate identification of fear facial expressions predicts prosocial behavior. Emotion, 7(2):239.

Peckham, A. D., McHugh, R. K., and Otto, M. W. (2010). A meta-analysis of the magnitude of biased attention in depression. Depression and Anxiety, 27(12):11351142.

Rush, A. J., Trivedi, M. H., Ibrahim, H. M., Carmody, T. J.,
Arnow, B., Klein, D. N., Markowitz, J. C., Ninan, P. T., Kornstein, S., and Manber, R. (2003). The 16-Item Quick Inventory of Depressive Symptomatology (QIDS), clinician rating (QIDS-C), and selfreport (QIDS-SR): a psychometric evaluation in patients with chronic major depression. Biological Psychiatry, 54(5):573-583.

Rutherford, M. D., Chattha, H. M., and Krysko, K. M. (2008). The use of aftereffects in the study of relationships among emotion categories. Journal of Experimental Psychology: Human Perception and Performance, 34(1):27-40.

Schenker, N. and Gentleman, J. F. (2001). On judging the significance of differences by examining the overlap between confidence intervals. The American Statistician, 55(3):182-186.

Schepman, K., Taylor, E., Collishaw, S., and Fombonne, E. (2012). Face emotion processing in depressed children and adolescents with and without comorbid conduct disorder. Journal of Abnormal Child Psychology, 40(4):583-593.

Series, P., Stocker, A. A., and Simoncelli, E. P. (2009). Is the homunculus "aware" of sensory adaptation? Neural Computation, 21(12):3271-3304.

Sheehan, D. V., Lecrubier, Y., Sheehan, K. H., Janavs, J., Weiller, E., Keskiner, A., Schinka, J., Knapp, E., Sheehan, M. F., and Dunbar, G. C. (1997). The validity of the Mini International Neuropsychiatric Interview (MINI) according to the SCID-P and its reliability. European Psychiatry, 12(5):232-241.

Soto, F. A. and Ashby, F. G. (2019). Novel representations that support rule-based categorization are acquired onthe-fly during category learning. Psychological Research, 83(3):544-566.

Soto, F. A., Vucovich, L., Musgrave, R., and Ashby, F. G. (2015). General recognition theory with individual differences: A new method for examining perceptual and decisional interactions with an application to face perception. Psychonomic Bulletin $\mathcal{F}$ Review, 22(1):88111.

Soto, F. A., Vucovich, L. E., and Ashby, F. G. (2018). Linking signal detection theory and encoding models to reveal independent neural representations from neuroimaging data. PLOS Computational Biology, 14(10):e1006470.

Soto, F. A., Zheng, E., Fonseca, J., and Ashby, F. G. (2017). Testing separability and independence of perceptual dimensions with general recognition theory: a tutorial and new R package (grtools). Frontiers in Psychology, $8: 696$

Surguladze, S. A., Young, A. W., Senior, C., Brébion, G., Travis, M. J., and Phillips, M. L. (2004). Recognition accuracy and response bias to happy and sad facial ex- 
pressions in patients with major depression. Neuropsychology, 18(2):212.

Wilkinson, P. O. and Goodyer, I. M. (2006). Attention diffi- culties and mood-related ruminative response style in adolescents with unipolar depression. Journal of Child Psychology and Psychiatry, 47(12):1284-1291. 


\section{Supplementary Online Material For: \\ A Computational Account of the Mechanisms Underlying Face Perception Biases in Depression}

Fabian A. Soto, Rochelle A. Stewart, Sanaz Hosseini, Jason Hays, \& Christopher G. Beevers

\section{Pilot Study}

A pilot study was performed to obtain psychometric curves useful to perform stimulus calibration for the main study. Identities were grouped into two stimulus sets (see Figure S1). Seven different morph sequences were created for each stimulus set, using the software JPsychMoph, going from one face to another in 10\% steps. The first sequence went from one neutral identity to the other, thus varying in identity but having no information about emotion. To create the other sequences, we first averaged the two identities in a stimulus set at each emotional expression. Then we created a sequence for each emotion, starting at neutral and ending in the target emotion, thus varying in emotion but having no information about identity. In sum, there were two stimulus sets, each composed of 3 morphing sequences each.

A different group of 20 participants was tested with each morphing sequence. Participants were first trained to discriminate the two extremes of the sequence for a total of 48 trials. This was followed by 50 blocks of testing, each involving 6 training trials with the extremes of the sequence and 9 testing trials with all the faces between the two extremes. Participants were excluded from the final analysis if their accuracy in the training trials did not reach $75 \%$ correct. The data from each participant was fitted to a signal detection theory model of the psychometric curve (Lesmes et al., 2015) using the R package quickpsy (Linares \& López-Moliner, 2016), and the average of the parameters was used to obtain a group psychometric curve. The resulting model includes a sensitivity psychometric function, in which $d$ ' is a function of level of morphing. This allowed us to choose any level of morphing that, on average, would produce a given value of $d$ ' when compared to the origin of the sequence.

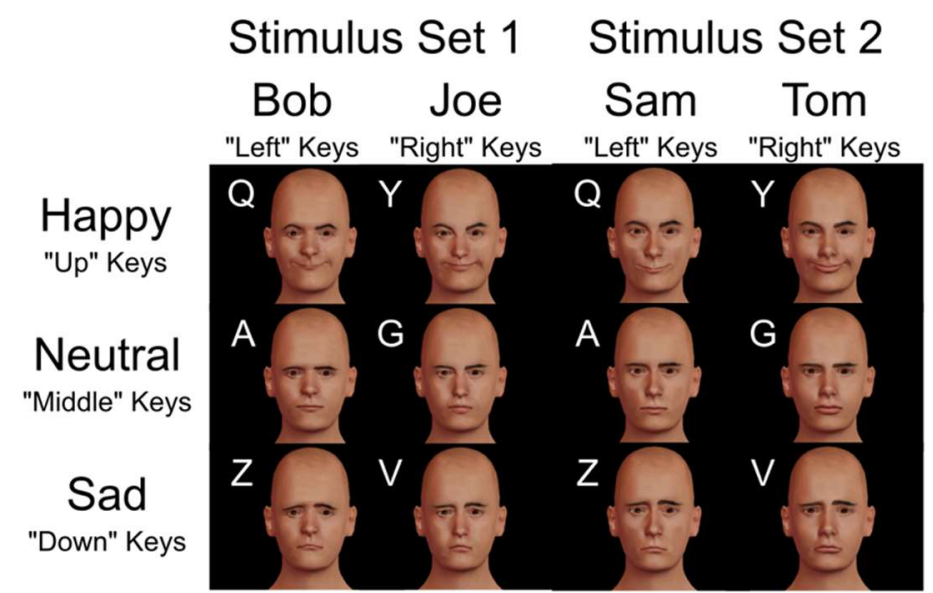

Figure S1. Renderings of original face models used for the main study (not actual stimuli shown), together with keys assigned to each combination of identity and expression in the identification tasks. 


\section{Supplementary Methods \\ Psychometric properties of the QIDS-SR.}

The QIDS-SR (Rush et al., 2003) is a 16-item self-report questionnaire that assesses the nine diagnostic symptom domains used to characterize a major depressive episode. The psychometric properties of the QIDS-SR have been studied extensively and are very good (Rush et al., 2003). For instance, across several large samples of adults with MDD, Cronbach's alpha ranged from .83 to .86 (Rush et al., 2003; Trivedi et al. (2004) and is unifactorial (Rush et al., 2003). Other work demonstrates that the QIDS-SR is sensitive to change with medications, psychotherapy, or somatic treatments (Rush et al., 2006). For instance, in 681 patients with chronic MDD assigned to 3 treatment groups (medication alone, medication and psychotherapy, psychotherapy alone), Rush et al. (2005) found that change in QIDS-SR was comparable to change measured by a standard depression symptom severity interview, the Hamilton Rating Scale for Depression (Hamilton, 1967).

\section{Training of research assistants applying the MINI.}

Research assistants took part in a training during which they learned interview skills, role-played interviews, and reviewed diagnostic criteria. After the workshop, they listened to interviews conducted by experienced researchers and had their initial screening interviews monitored for fidelity. The research assistant had a bachelor's degree in psychology and past experience with clinical interviewing. After the training, a licensed clinical psychologist with extensive experience with diagnostic assessment, who was not a rater for any of the study interviews, monitored their screening interviews. The initial interviews were rated by study assessors and the licensed clinical psychologist. Any disagreements were discussed and a consensus diagnosis was determined. Once the assessor achieved strong concordance with the experienced rater, the rater then randomly checked $10 \%$ of the subsequent interviews. Agreement for current and lifetime MDD was excellent (kappas $=1.00,0.82$, respectively).

\section{Modeling Details \\ GRT-wIND Model.}

Data from the identification experiments in our study was fitted to General Recognition Theory with INdividual Differences, or GRT-wIND (Soto, Vucovich, Musgrave, \& Ashby, 2015). GRT-wIND is a multi-level version of general recognition theory, with "group parameters" representing aspects of perceptual processing assumed to be common across all participants in a particular study condition, and "individual parameters" representing aspects of perceptual processing assumed to be idiosyncratic to each participant, particularly the level of attention to the task and dimensions, and decisional strategies used to solve the task. GRT-wIND was used because it is at the moment the only GRT model known to avoid parameter identifiability and over-fitting problems common in GRT modeling of individual data (Silbert \& Thomas, 2013; Soto et al., 2015).

Each experiment in our report was sub-divided into three different $2 \times 2$ identification tasks, with stimuli that could display one of two levels of identity and one of two levels of emotional expression. Each of these four stimuli is indexed by $s$, and its perceptual representation in the model was captured by a bivariate Gaussian distribution, described by a mean vector: 


$$
\boldsymbol{\mu}_{s}=\left[\begin{array}{l}
\mu_{s, x} \\
\mu_{s, y}
\end{array}\right]
$$

and a covariance matrix:

$$
\boldsymbol{\Sigma}_{s}=\left[\begin{array}{cc}
\sigma_{s, x}^{2} & \rho_{s} \sigma_{s, x} \sigma_{s, y} \\
\rho_{s} \sigma_{s, x} \sigma_{s, y} & \sigma_{s, y}^{2}
\end{array}\right](2)
$$

where $x$ represents the perceptual dimension of identity, $y$ represents the perceptual dimension of emotional expression, $\sigma$ is a standard deviation parameter and $\rho$ is a correlation parameter. We set $\boldsymbol{\mu}_{1}=\left[\begin{array}{ll}0 & 0\end{array}\right]$ to fix the position of the model in the two-dimensional space of $x$ and $y$, and all $\sigma=1$ to fix the scale of the model and avoid identifiability problems that are known to arise between means and variances in signal detection theory models (Silbert \& Thomas, 2017). Together, all these parameters describing bivariate perceptual distributions comprise the "group parameters" in GRT-wIND.

GRT-wIND also includes several individual parameters, which allow to capture individual differences in performance of the identification task. Two parameters describe the level of attention that participant $k$ has for the task and each stimulus dimension. The parameter $\kappa_{k}$ represents the level of attention that participant $k$ pays to the task as a whole, with higher values decreasing perceptual noise in the model and producing better discrimination along both dimensions. On the other hand, the parameter $\lambda_{k}$ represents the level of selective attention that participant $k$ pays to each dimension, with a value of 0.5 representing equal attention to both dimensions, a value higher than 0.5 representing more attention to identity, and a value lower than 0.5 representing more attention to emotional expression. These parameters modify the covariance matrix for each participant in the following way:

$$
\boldsymbol{\Sigma}_{s k}=\left[\begin{array}{cc}
\frac{\sigma_{s, x}^{2}}{\kappa_{k} \lambda_{k}} & \rho_{s} \frac{\sigma_{s, x} \sigma_{s, y}}{\sqrt{\kappa_{k}^{2} \lambda_{k}\left(1-\lambda_{k}\right)}} \\
\rho_{s} \frac{\sigma_{s, x} \sigma_{s, y}}{\sqrt{\kappa_{k}^{2} \lambda_{k}\left(1-\lambda_{k}\right)}} & \frac{\sigma_{s, y}^{2}}{\kappa_{k}\left(1-\lambda_{k}\right)}
\end{array}\right],
$$

A second set of individual parameters describe two linear decision bounds assumed to be used by each participant to divide the perceptual space into four response areas. Each decision bound can be written as a discriminant function:

$$
h_{d k}(X, Y)=b_{d k, x} X+b_{d k, y} Y+c_{d k}
$$

where $d$ indexes a particular discrimination (e.g., $d=$ neutral/happy, or $d=B o b / J o e$ ), and $X$ and $Y$ represent specific values in the dimensions $x$ and $y$, respectively. One parameter in this equation is always fixed to one and the other two parameters control the slope and position of the decision bound. 
GRT-wIND Model Parameters Estimation. Assume that $R_{j}$, with $j$ going from 1 to 4 , represents a particular response combination in an identification task, such as "Happy Joe", "Neutral Joe", "Happy Bob", and "Neutral Bob". Then the probability of each response combination given the presentation of stimulus $s$, or $P\left(R_{j} \mid s\right)$, is the area of the Gaussian distribution representing $s$ that falls within the response region assigned to $R_{j}$. Such response probabilities can be used to compute the likelihood of a given set of parameters, given the data. We estimated the model parameters for each condition in our study by maximizing the loglikelihood of the model:

$$
L L(\boldsymbol{\theta})=\sum_{k} \sum_{s} \sum_{j} r_{k s j} \log P_{k}\left(R_{j} \mid s, \boldsymbol{\theta}\right)
$$

where $r_{k s j}$ represents the frequency with which participant $k$ reported the response combination $R_{j}$ when presented with stimulus $s$, and $P_{k}\left(R_{j} \mid s, \boldsymbol{\theta}\right)$ is the probability of such an event obtained from the model with a given set of parameters $\theta$.

Computation of Perceptual and Decisional Processing Measures. GRT is different from other approaches in that measures derived from the obtained model are more meaningful than the actual model parameters. For example, other models commonly used in computational psychiatry are the drift decision model and reinforcement learning models. In the case of those models, parameters estimated from data can be directly interpretable as representing some perceptual or cognitive process, such as the rate of accumulation of sensory evidence or the speed of learning. On the other hand, parameter values in GRT are more meaningfully interpreted in relation to other parameter values. For example, if we have two perceptual distributions for "Happy Bob" and "Neutral Bob," their position parameters represented in the model by $\boldsymbol{\mu}_{s}$ are not very meaningful by themselves; what matters is their distance along a particular dimension. This can be seen more generally in Figure 1 of the main text, where all measures of interest are actually some form of distance. In this section, we explain how we computed distance measures from the parameters obtained from GRT-wIND.

Perceptual Discriminability. Perceptual discriminability is measured in signal detection theory through sensitivity, or the standardized distance between perceptual distributions along a particular dimension (see Figure 1a). When variances are set to one, as in our model, the sensitivity of the discrimination between stimuli $s_{1}$ and $s_{2}$ along dimension $x$ is computed in the following way:

$$
d_{x}^{\prime}\left(s_{1}, s_{2}\right)=\left|\mu_{s_{1}, x}-\mu_{s_{2}, x}\right|
$$

Threshold Bias. One way to measure bias in SDT is by computing the distance between a given threshold and the position of an optimal bound. In GRT, where distributions are positioned in a two-dimensional space and thresholds are replaced by bounds (see Equation 4), it is less straightforward to determine a reference point from which the bias should be computed. Here, we took the simple approach of computing the center of the model's representation and use it as a reference point: 


$$
\overline{\boldsymbol{\mu}}=\frac{1}{4} \sum_{s} \boldsymbol{\mu}_{s}
$$

We computed bias in dimension $x$ as the deviation of a bound's position in that dimension from the center of the representation. We replace the center coordinates from Equation 7 into the discriminant function from Equation 4, but we include a term to $\beta_{d k, x}$, to determine to what extent we would have to shift $\bar{\mu}$ along the $x$ axis so that it would be on the bound (when the discriminant function would result in a value of zero):

$$
\begin{aligned}
& 0=b_{d k, x}\left(\bar{\mu}_{x}+\beta_{d k, x}\right)+b_{d k, y} \bar{\mu}_{y}+c_{d k} \\
& \beta_{d k, x}=-\left(\bar{\mu}_{x}+\frac{b_{d k, y} \bar{\mu}_{y}+c_{d k}}{b_{d k, x}}\right)
\end{aligned}
$$

An analogous equation could be obtained for $\beta_{d k, y}$, but we only analyzed thresholds for the emotion task.

Perceptual Separability. The distance between two distributions that share a value in a particular relevant dimension (e.g., emotional expression), but different values in an irrelevant dimension (e.g., identity), provides a numerical estimate of deviations from perceptual separability. One way to measure this distance is through the L1 norm:

$$
L 1=\int\left|p_{1}(z)-p_{2}(z)\right| d z,
$$

where $p_{1}$ and $p_{2}$ represent the marginal distributions of values along the relevant dimension for two different levels ( 1 and 2) of the irrelevant dimension. The $L 1$ distance is represented in Figure $1 \mathrm{~b}$ by the areas shaded in blue and red. To compute $L 1$, we obtained 100 evenly spaced values of the dimensional variable $z$, starting five standard deviations to the left of the smaller of the two means of $p_{1}$ and $p_{2}$, and ending five standard deviations to the right of the larger of those two means. Let $z_{k}$ with $k=1,2, \ldots 100$ represent these discrete values of the relevant dimension $z$. then an estimate of the of the summed $L 1$ distances representind deviations from perceptual similarity can be computed in the following way:

$$
L 1_{d}^{G}=\sum_{m} \sum_{k}\left|p_{m 1}\left(z_{k}\right)-p_{m 2}\left(z_{k}\right)\right|,
$$

Where $d$ stands for the relevant discrimination for which perceptual separability is being computed, $G$ stands for $g l o b a l$ as this is a measure that adds multiple $L 1$ estimates (i.e., the sum of the blue and red areas in Figure 1b), and $m$ indexes the levels of the relevant dimension.

Decisional separability. As explained in the main text, we computed deviations from decisional separability as an angular distance, as shown in Figure 1b. More precisely, the GRTwIND model included a single bound for each dimension, represented by the discriminant function in Equation 4. From the estimated parameters of this discriminant function, a measure of deviations from decisional separability is the clockwise rotation (in degrees) of the decision bounds from orthogonality with respect to the dimension they classify. For example, in the case of the decision bound for the emotion discrimination, which was represented in the model along the $x$ axis, our measure of the decisional separability is the following: 


$$
\varphi_{k, \text { emotion }}=-\operatorname{atan}\left(b_{d k, y}\right) \frac{180}{\pi} .
$$

Similarly, in the case of the decision bound for the identity discrimination, which was represented in the model along the $y$ axis, the measure is the following:

$$
\varphi_{k, \text { identity }}=\operatorname{atan}\left(b_{d k, x}\right) \frac{180}{\pi} .
$$

Both measures where averaged across participants (e.g., $\bar{\varphi}_{\text {emotion }}=\sum_{k} \varphi_{k \text {,emotion }}$ ) to report a single global measure of deviations from decisional separability in Table 1 from the main manuscript, but individual measures are also reported below in Figures S10 and S11.

Confidence Intervals on Estimated Model Parameters and Measures. To obtain confidence intervals on the estimated model parameters we used a parametric bootstrap procedure (Carpenter \& Bithell, 2000; Good, 2006). We used a parametric rather than a non-parametric bootstrap procedure because (1) our analysis assumes a model for the data distribution, so in this case the parametric bootstrap is appropriate (Carpenter \& Bithell, 2000), and (2) using the nonparametric bootstrap with small sample sizes such as ours leads to imprecise confidence intervals (Good, 2006). At each step of the bootstrap, a simulated data set was sampled from the fitted GRT-wIND model, and was used to fit a new model to the simulated data. For each parameter, this results in an empirical distribution function of parameter values, which was used to directly obtain 95\% confidence intervals using a simple quantile procedure. Similarly, we obtained confidence intervals on values derived from parameter estimates, such as the $d$ ' and $L 1$ statistics, by computing such values from the bootstrapped parameter estimates and obtaining an empirical distribution function for each statistic.

\section{Computational Model of Perceptual Biases in Depression.}

We assume that the intensity of an expression can have positive values (e.g., happy) and negative values (e.g., anti-happy), with a value of zero being neutral. The model of face expression encoding consists of two channels with opponent stimulus preference: the first channel responds maximally to high negative values of expression (e.g., extremely anti-happy, see black curve in Figure 2a of the main manuscript), while the second channel responds maximally to high positive values of expression (e.g., extremely happy, see red curve in Figure 2a of the main manuscript). The response of each channel as a function of expression intensity is characterized by a logistic function (see Figure 2a in main manuscript):

$$
f_{c}(s)=a_{c}^{\max } \frac{1}{1+\exp \left(q_{c}\left(s_{c}-s\right)\right)}
$$

where $a_{c}^{\max }$ represents the maximum response for channel $c, s_{c}$ is a position parameter that represents the value of expression intensity where the curve is midway between zero and $a_{c}^{\max }$, and $q_{c}$ is a parameter determining the slope of the logistic curve.

Our model of expression encoding in healthy people has parameters $a_{c}^{\max }=20$ for both channels, $s_{c}=-0.4$ and $q_{c}=-3$ for the first channel, and $s_{c}=0.4$ and $q_{c}=3$ for the second 
channel. Our model of expression encoding in people with depression is identical, but with suppressed maximum response $a_{c}^{\max }=10$ for the second channel.

The predictions of our model depend critically on the parameters chosen for the tuning functions. Susilo, McKone and Edwards (2010) identify three candidate shapes for the response functions underlying opponent coding. One choice are linear functions, which can be obtained in the range of stimuli used in our simulations by setting $q_{c}=1$. A more common choice in the literature is one in which S-shaped functions are both centered at the midpoint of the stimulus dimension, which in our case would be obtained with $s_{c}=0$. Finally, it is possible to have a "separated-S" model, in which two S-shaped functions are displaced from the midpoint of the stimulus dimension. Out of these three options, only the "separated-S" model could reproduce the results obtained in our main study $\left(s_{c}= \pm 0.4\right)$.

Our model assumes that the response of each channel $a_{c}$ is noisy, with the random component described by a Poisson distribution, independently for each channel:

$$
P\left(a_{c} \mid s\right)=\frac{f_{c}(s)^{a_{c}} \exp \left(-f_{c}(s)\right)}{a_{c} !}
$$

When a face stimulus is presented to the model, the output of the model is a vector of channel responses a, which implicitly encodes information about the expression intensity s. This output can be used by an observer to determine what stimulus was more likely to have been presented, and that value can be used to give behavioral responses in a psychophysical task. As in previous computational research (e.g., Deneve, Latham, \& Pouget, 1999; May \& Solomon, 2015; Paradiso, 1988; Series, Stocker, \& Simoncelli, 2009), we assume an optimal observer that computes an estimate of expression with value $\hat{s}$ by maximizing the log-likelihood of the channel output as a function of the stimulus:

$$
\ln p(\boldsymbol{a} \mid s) \propto \sum_{c} a_{c} \ln a_{c}^{\max }-\ln \left(1+\exp \left(q_{c}\left(s_{c}-s\right)\right)\right)-\sum_{c} \frac{a_{c}^{\max }}{1+\exp \left(q_{c}\left(s_{c}-s\right)\right)}
$$

Because the channel response pattern a includes channel noise, repeated presentations of the same stimulus produce different values of $\mathbf{a}$, and $\hat{s}$ in turn is randomly distributed. The random distribution of $\hat{s}$ corresponds to the distribution of perceptual evidence assumed by signal detection theory, which allows to link the opponent-channel observer model to measures such as $d^{\prime}$. We used Monte Carlo simulation to estimate the mean and standard deviation of the distribution of estimates $\hat{s}$ after the presentation of a stimulus value $s$. The values of $s$ ranged from -1 to +1 in 0.1 steps. For each of such values, the stimulus $s$ was presented to the model and an estimate $\hat{s}$ was obtained. This process was repeated 3,000 times, and the mean $\mu_{\hat{s}}$ and standard deviation $\sigma_{\hat{s}}$ of the resulting values of $\hat{s}$ were computed. Such values were used to compute the $d^{\prime}$ values presented in Figure $2 \mathrm{c}$ according to the following equation:

$$
d^{\prime}=\frac{\mu_{\hat{s}_{1}}-\mu_{\hat{s}_{2}}}{\sqrt[2]{.5\left(\sigma_{\hat{s}_{1}}^{2}+\sigma_{\hat{s}_{2}}^{2}\right)}}
$$


The overall channel activity presented in Figures $2 \mathrm{~d}$ and $2 \mathrm{e}$ of the main manuscript were computed in the following way:

$$
A=\sum_{c} f_{c}(s)
$$

where $f_{c}(s)$ is defined by Equation 13 .

\section{Supplementary Results}

Number of participants included in each analysis and their accuracy in the identification task.

SDT analyses require that performance be well-above chance and below perfect, so each separate analysis included only data from participants who had performance above .4 and below .95 percent correct. Table S1 reports the number of participants included in each analysis per group, as well as the mean and standard deviation of their proportion of correct responses.

\section{Table S1. Number of participants included in each analysis (N), and mean and standard} deviation (S.D.) of their proportion of correct responses, separated by group.

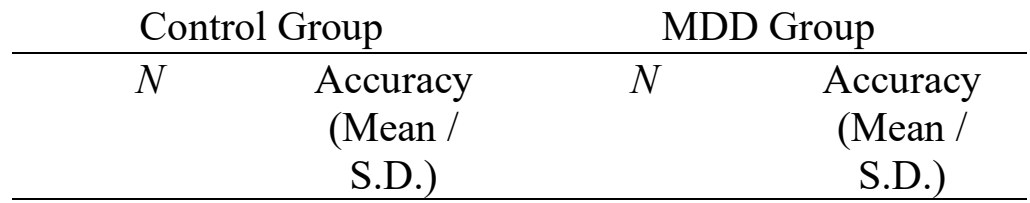

\section{Session 1 (Stimulus Set 1)}

Neutral vs. Sad

Neutral vs. Happy

Sad vs. Happy

Session 2 (Stimulus Set 2)

Neutral vs. Sad

Neutral vs. Happy

Sad vs. Happy
25

28

29

23

27

25
$.72 / .12$

$.75 / .15$

$.72 / .17$

$.76 / .13$

$.79 / .14$

$.79 / .14$
26

27

27

$.73 / .12$

$.74 / .15$

$.79 / .12$

Session 3 (Stimulus Set 1 Ambiguous)

Neutral vs. Sad

Neutral vs. Happy

Sad vs. Happy

25

24

26
$.71 / .15$

$.73 / .15$

$.72 / .14$
28

26

26
$.75 / .13$

$.77 / .10$

$.79 / .13$

It is important to highlight that the averaged proportion of correct responses shown in Table $\mathrm{S} 1$ are relatively uninformative about the results of the main model-based analysis. The model is fitted directly to individual confusion matrices, which provide information about a specific participant's perceptual and decisional parameters. 
Figures S2-S4 show average confusion matrices separately for different emotion discrimination tasks. Within each figure, confusion matrices are plotted separately for each session and group. The pattern of correct responses and confusion errors shown in Figures S2-S4 are closer to what our model uses to extract information about its perceptual and decisional parameters. However, these figures show averaged data that cannot truly display the complex patterns of results captured by the model in the individual data. It is a well-known fact in computational modeling of behavior that average data may produce incorrect data patterns and model inferences (e.g., Ashby, Maddox, \& Lee, 1994), so the average confusion matrices shown in Figures S2-S4 should be interpreted with caution, and as less informative than the results of our model-based analysis presented in the main manuscript. 


\section{Neutral vs. Sad}
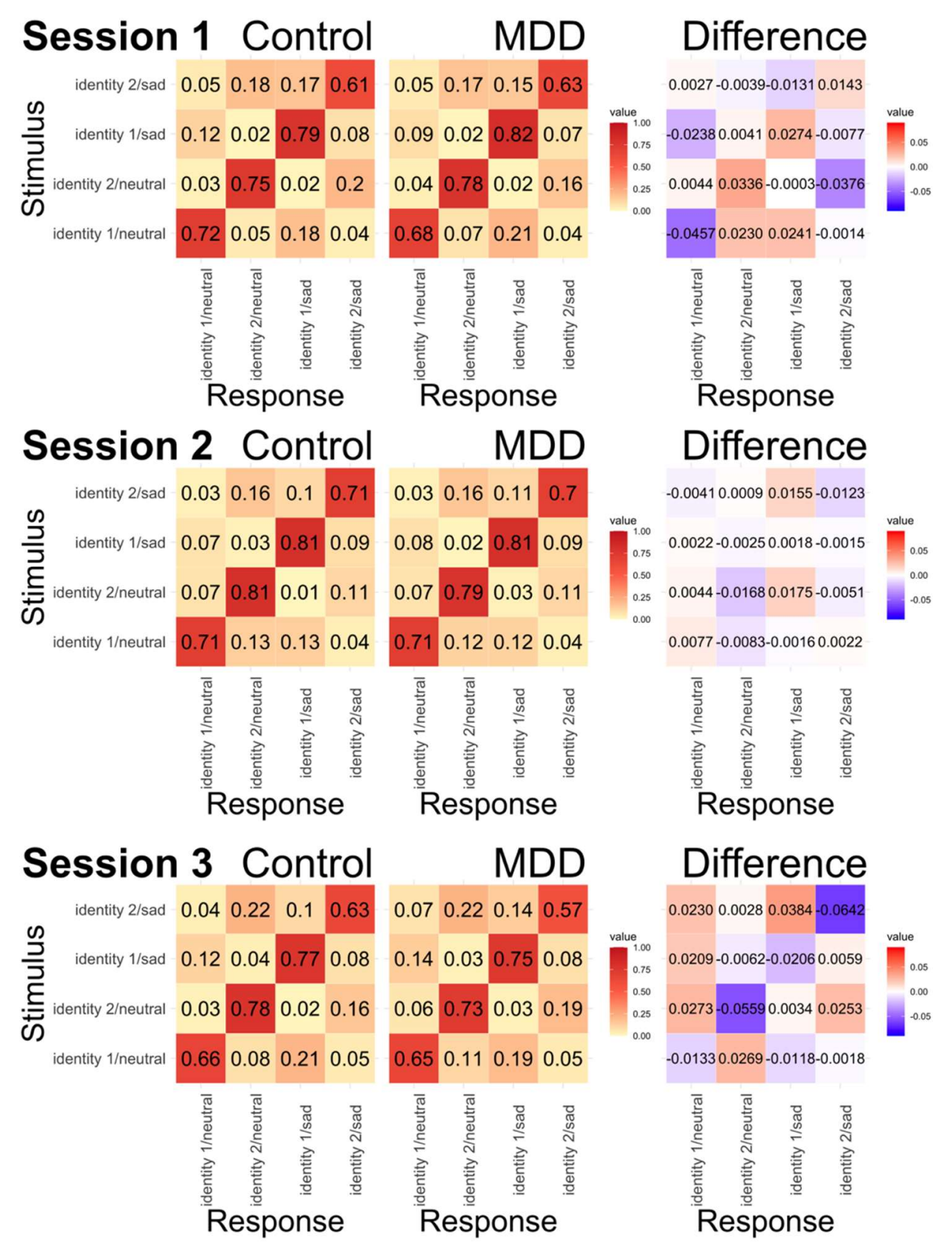

Figure S2. Average confusion matrices for the neutral vs. sad discrimination task. In the two first columns of matrices, values in each cell correspond to the proportion of responses of each type (columns) given to a particular stimulus (rows). Lower values are yellow, and higher values are red. The last column of matrices (labeled "Difference") represents the MDD confusion matrix minus the Control confusion matrix. Red cells represent higher scores for MDD, blue cells represent higher scores for Control. 


\section{Neutral vs. Happy}

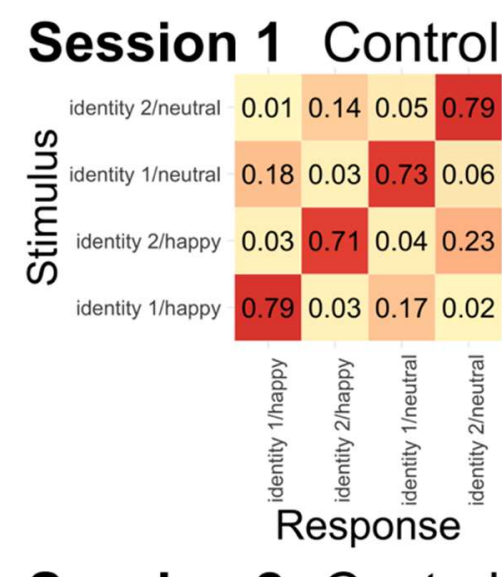

Session 2 Control
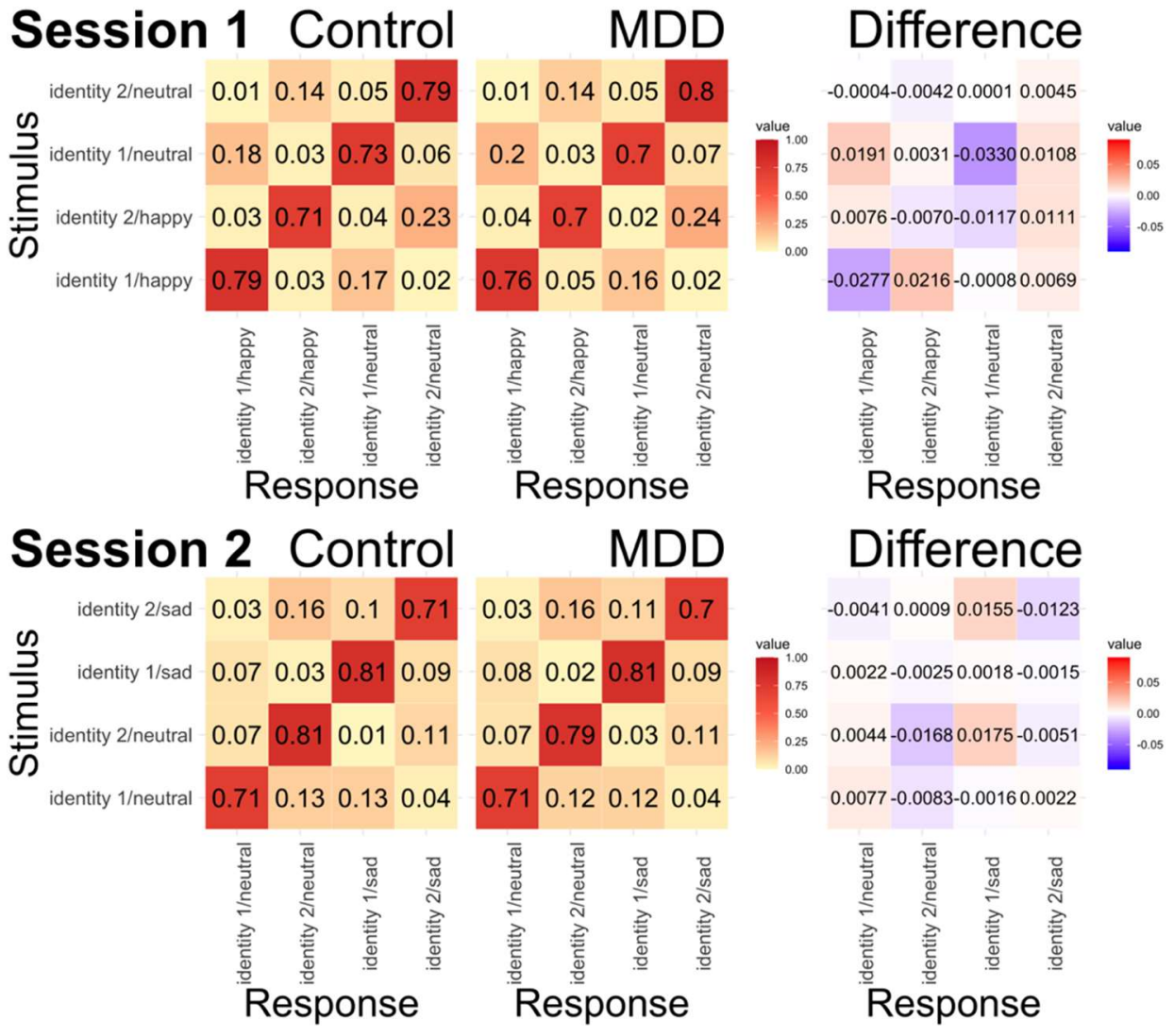

\section{Difference}

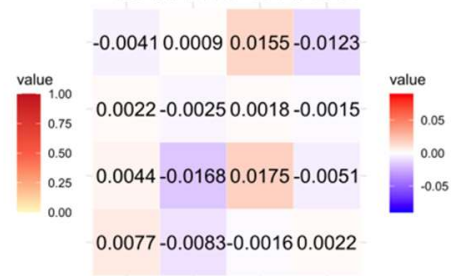

\section{Session 3 Control}
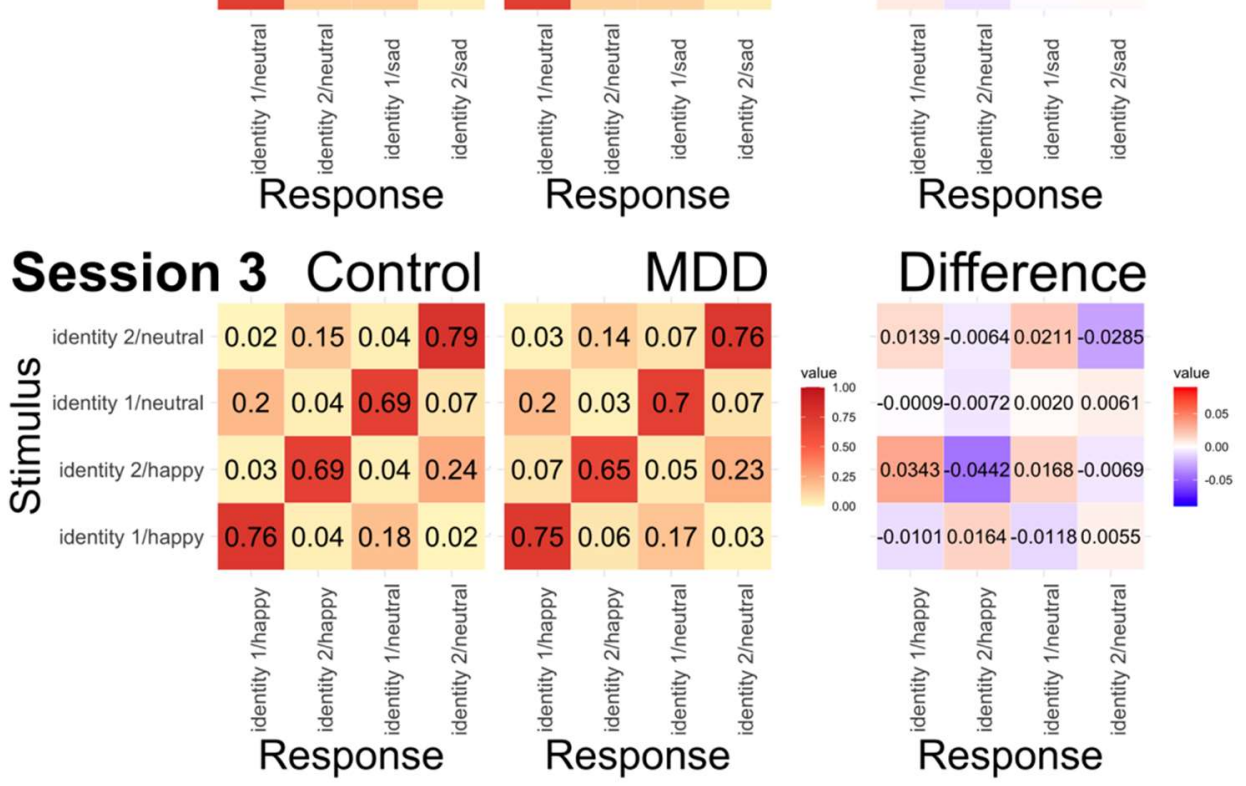

Figure S3. Average confusion matrices for the neutral vs. happy discrimination task. In the two first columns of matrices, values in each cell correspond to the proportion of responses of each type (columns) given to a particular stimulus (rows). Lower values are yellow, and higher values are red. The last column of matrices (labeled "Difference") represents the MDD confusion matrix minus the Control confusion matrix. Red cells represent higher scores for MDD, blue cells represent higher scores for Control. 


\section{Sad vs. Happy}
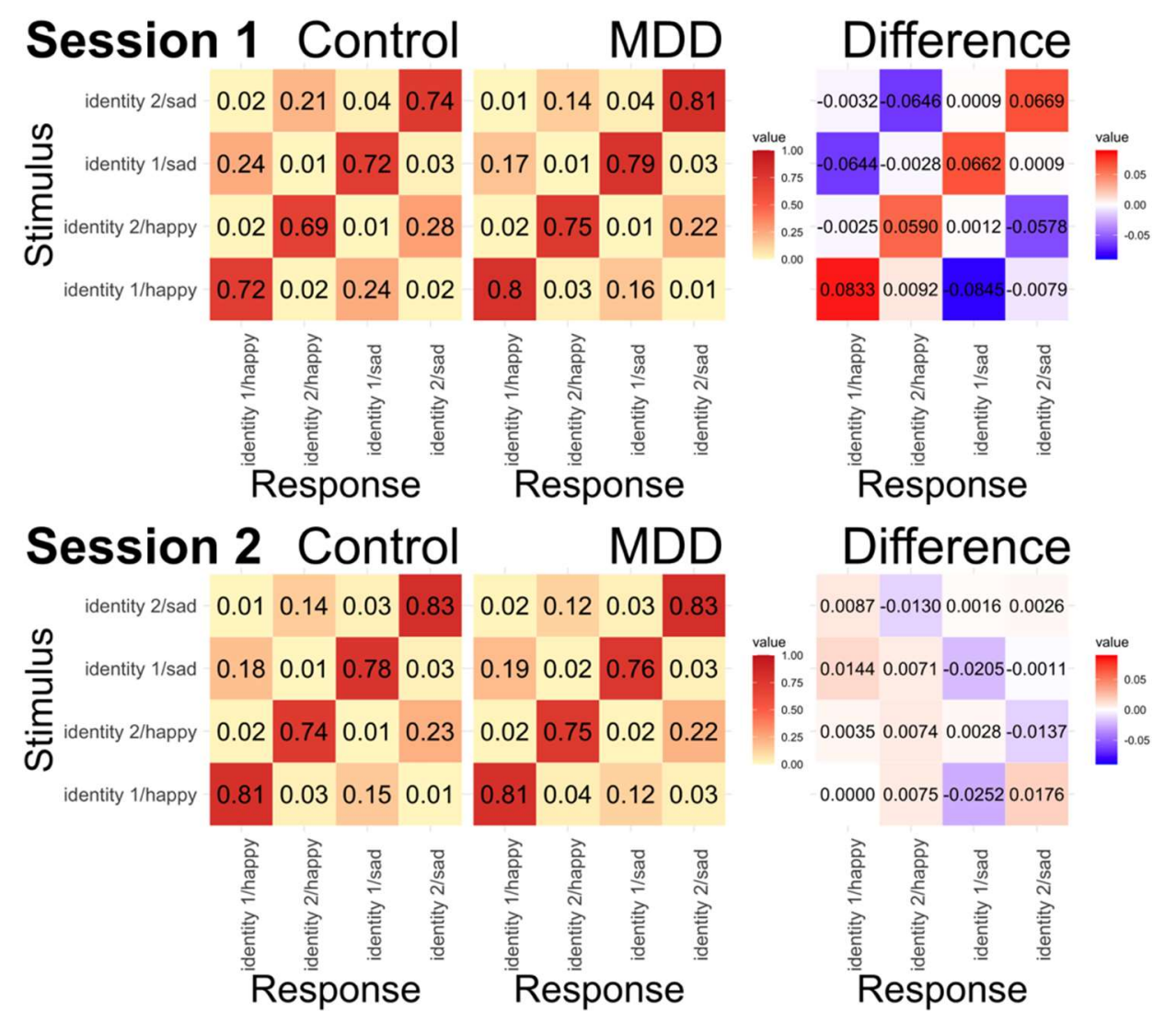

\section{Session 3 Control}

MDD
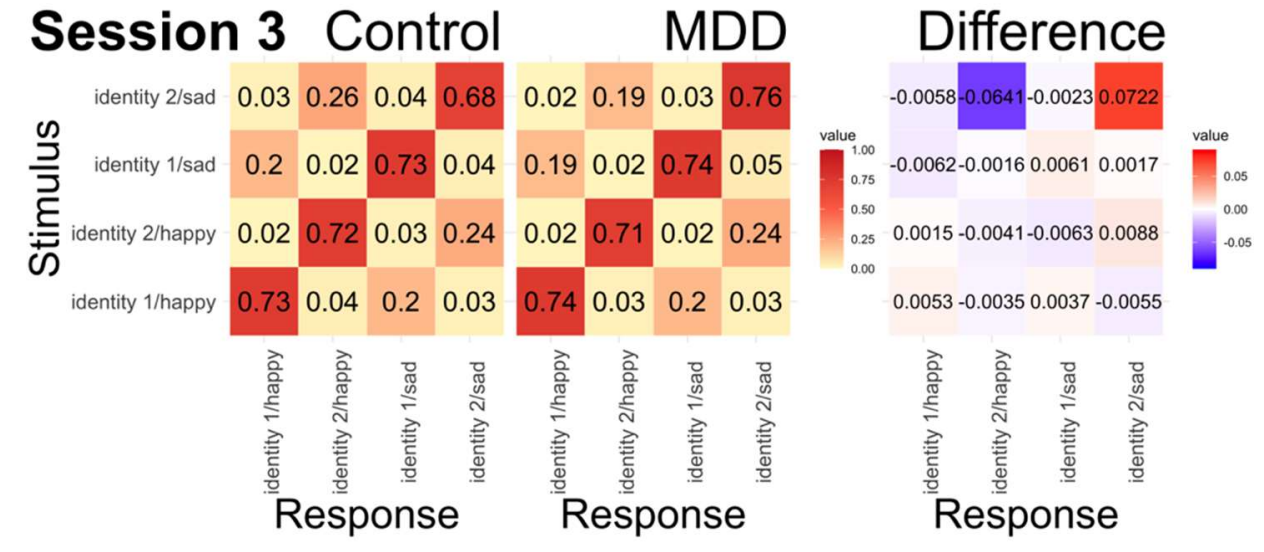

Figure S4. Average confusion matrices for the sad vs. happy discrimination task. In the two first columns of matrices, values in each cell correspond to the proportion of responses of each type (columns) given to a particular stimulus (rows). Lower values are yellow, and higher values are red. The last column of matrices (labeled "Difference") represents the MDD confusion matrix minus the Control confusion matrix. Red cells represent higher scores for MDD, blue cells represent higher scores for Control. 


\section{Measures of Model Fit}

Table S2 reports measures of model fit for the model-based analyses presented in the main manuscript. Although the main measure used to fit the model to data was the log-likelihood, this is difficult to interpret by itself. For this reason, we also computed a coefficient of determination $R^{2}$ by obtaining predicted probabilities of different responses from the final fitted model, correlating them with the observed response proportions from participants, and squaring the results. The interpretation of $R^{2}$ is straightforward as the proportion of the variance of observed response proportions that is explained by the model.

Table S2. Measures of fit of the GRT-wIND model to the different data sets obtained in our study.

\begin{tabular}{ccc}
\multicolumn{2}{c}{ Control Group } & \multicolumn{2}{c}{ MDD Group } \\
\hline $\begin{array}{c}\text { Log- } \\
\text { likelihood }\end{array}$ & $R^{2}$ & $\begin{array}{c}\text { Log- } \\
\text { likelihood }\end{array}$ \\
\hline
\end{tabular}

Session 1 (Stimulus Set 1)

Neutral vs. Sad

$-7,101.36 \quad .9876$

$-6,953.63$

.9921

Neutral vs. Happy

$-7,091.86$

.9924

$-7,091.06$

.9947

Sad vs. Happy

$-6,773.52$

.9816

$-6,156.59$

.9944

Session 2 (Stimulus Set 2)

Neutral vs. Sad

$$
-6,252.97
$$

$-5,997.85$

.9901

$-7,817.05$

.9937

Neutral vs. Happy

$-5,256.55$

.9945

$-6,540.94$

.9902

Sad vs. Happy

.9966

$-5,825.85$

.9914

Session 3 (Stimulus Set 1 Ambiguous)

Neutral vs. Sad

Neutral vs. Happy

Sad vs. Happy

$\begin{array}{llll}-7,588.87 & .9909 & -8,519.58 & .9867 \\ -6,575.48 & .9944 & -8,813.41 & .9916 \\ -6,963.05 & .9902 & -7,426.06 & .9925\end{array}$

\section{Detailed Results of Model-Based Analyses}

Detailed results are shown in Figures S1-S6, each sub-divided into three panels showing results from a different session. Because all these figures have the same structure, it is useful to provide a general explanation of that structure.

The top panel shows results from session one, with the unambiguous "Bob vs. Joe" stimuli. Results in this panel provide an initial answer to the question of whether MDD influences perceptual processing of face emotion. The middle panel shows results from session two, with the unambiguous "Sam vs. Tom". Results in this panel indicate whether changing face identities (with respect to session one) in turn modifies the effect of MDD on perceptual processing of face emotion. The bottom panel shows results from session three, with the 
ambiguous "Bob vs. Joe". Results in this panel indicate whether increasing ambiguity in the faces (with respect to session one) increases the effect of MDD on perceptual processing of face emotion.

Each panel shows the results separated by group (Control in red, and MDD in green) and task (in different columns: Neutral vs. Sad, Neutral vs. Happy, and Happy vs. Sad). The top of each panel depicts the actual parameters obtained from the best-fitting model (i.e., individual $d$ ' values in Figure 2 and $L 1$ distances between perceptual distributions in Figures 3 and 4). The bottom of each panel depicts summary statistics (i.e., mean $d$ ' in Figure 2 and sum of $L 1$ distances in Figures 3 and 4) as black dots, their 95\% confidence interval as black lines, and their bootstrap distribution (red and green areas). We can use the overlap between these confidence intervals to determine whether there were reliable differences between MDD and Control groups in an aspect of perceptual processing. 
Does MDD influence the perceptual discriminability of emotion?

ONLY FOR HAPPINESS. Discriminability of happiness was slightly impaired in MDD.

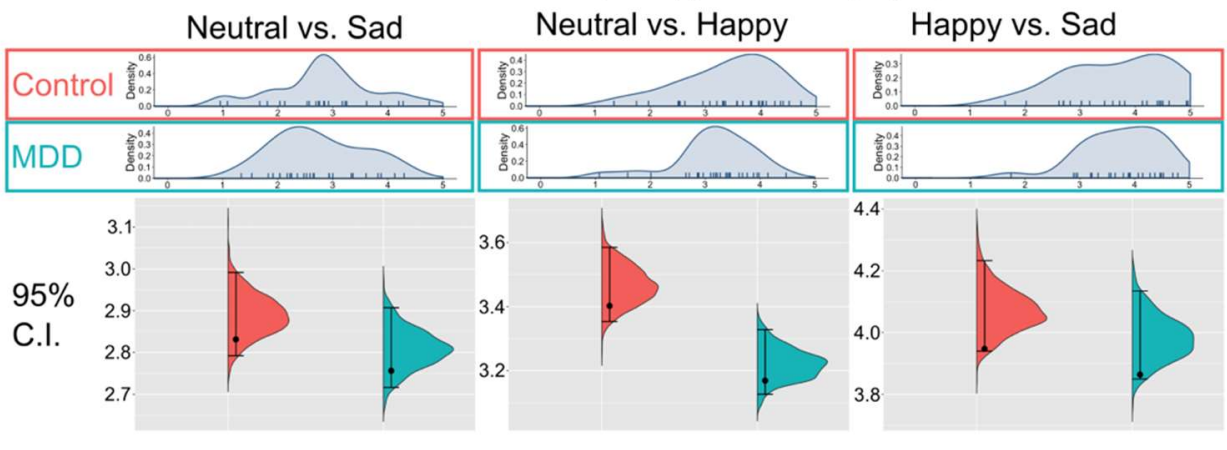

Does the result depend on specific face identities?

NO. The pattern of results remained the same when the identities were changed.

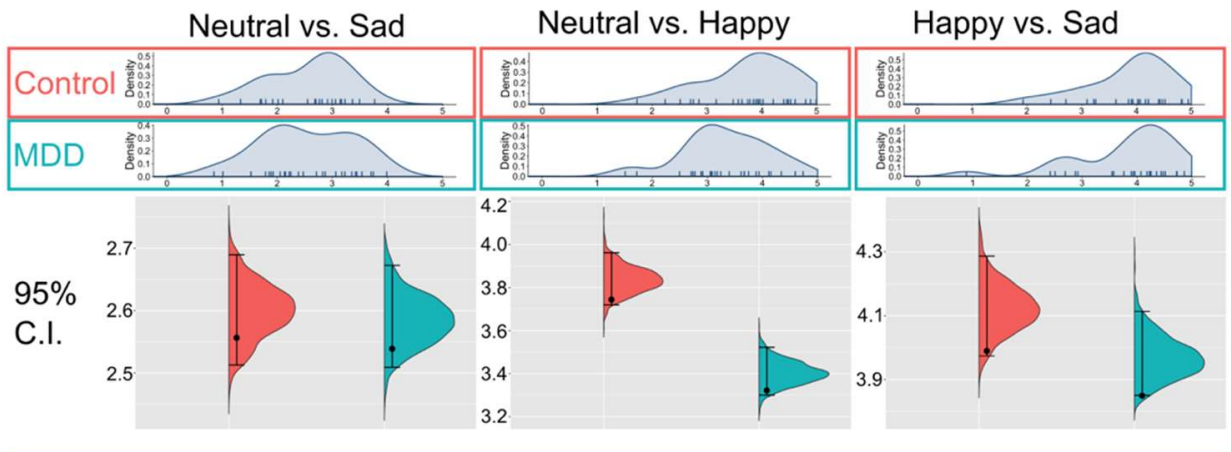

Does the result depend on ambiguity in the face information?

YES. Ambiguity produced poorer discriminability for MDD across all emotion pairs.

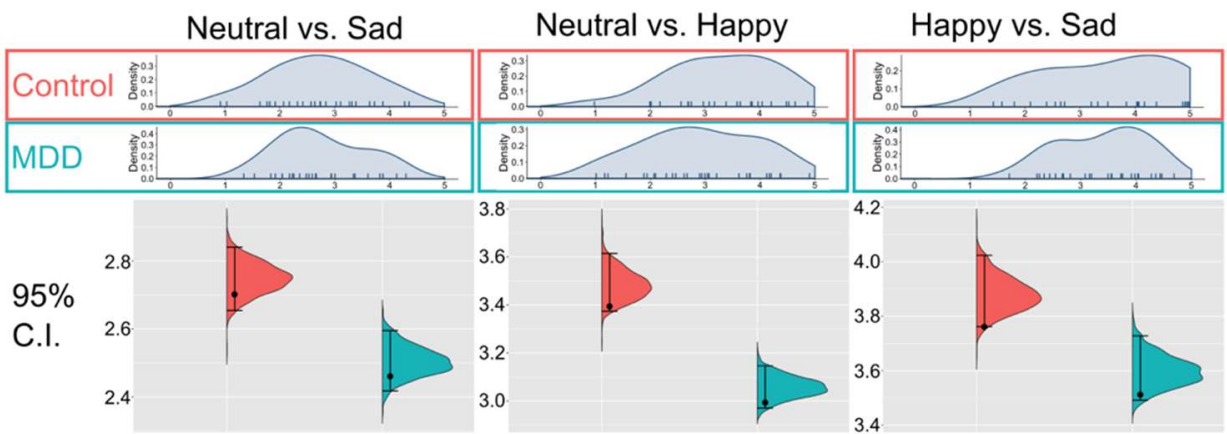

Figure S5. Detailed results on the analysis of perceptual discriminability of emotion. The blue distributions at the top of each panel represent the distribution of d'values (tick marks represent individual values). The y-axis in the CI plots represents mean d'. See main manuscript for more details. 
Does MDD influence the perceptual separability of emotion?

NO. Deviations from perceptual separability were similar across groups for all emotions

Neutral vs. Sad

Does the result depend on specific face identities?

ONLY FOR SADNESS, where deviations from perceptual separability were stronger for MDD.

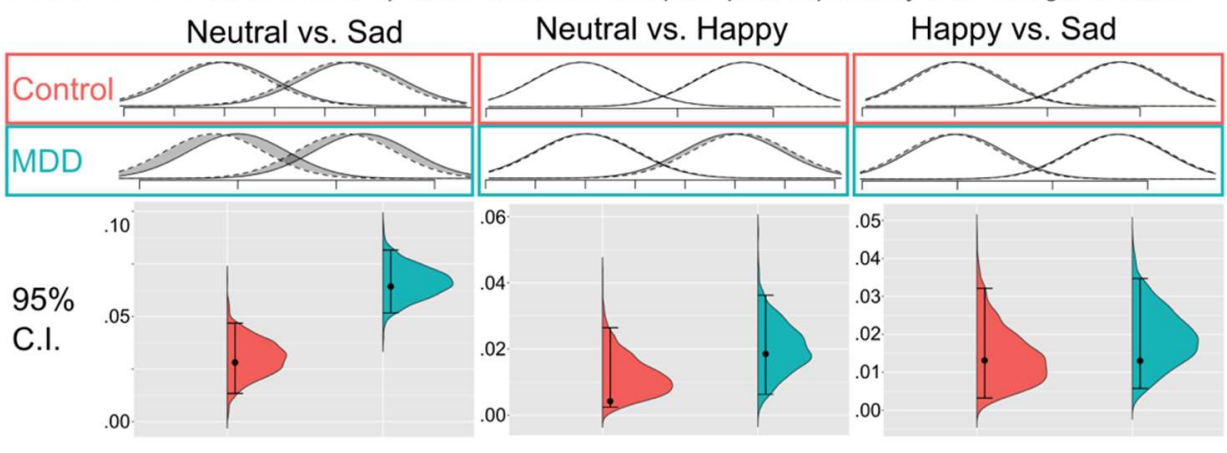

Does the result depend on ambiguity in the face information?

NO. Results did not change when ambiguity was increased.

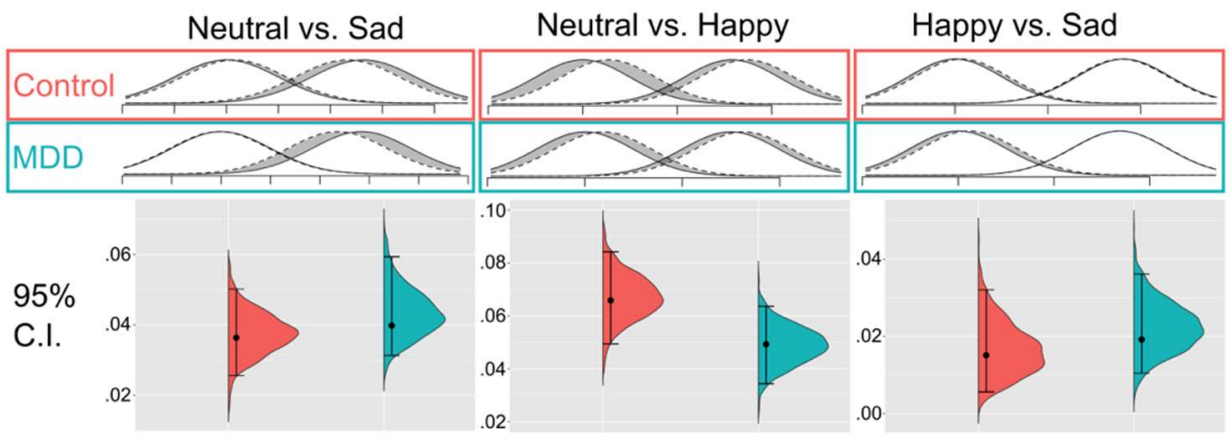

Figure S6. Detailed results on the analysis of perceptual separability of emotion. Perceptual distributions recovered from the model are shown in the top row of each panel, with solid curves representing one identity and dotted curves the other identity. The shaded area represents the LI distance between perceptual distributions. See more details in main manuscript. 
Does MDD influence the perceptual separability of identity?

NO. Deviations from perceptual separability were similar between groups for all emotions

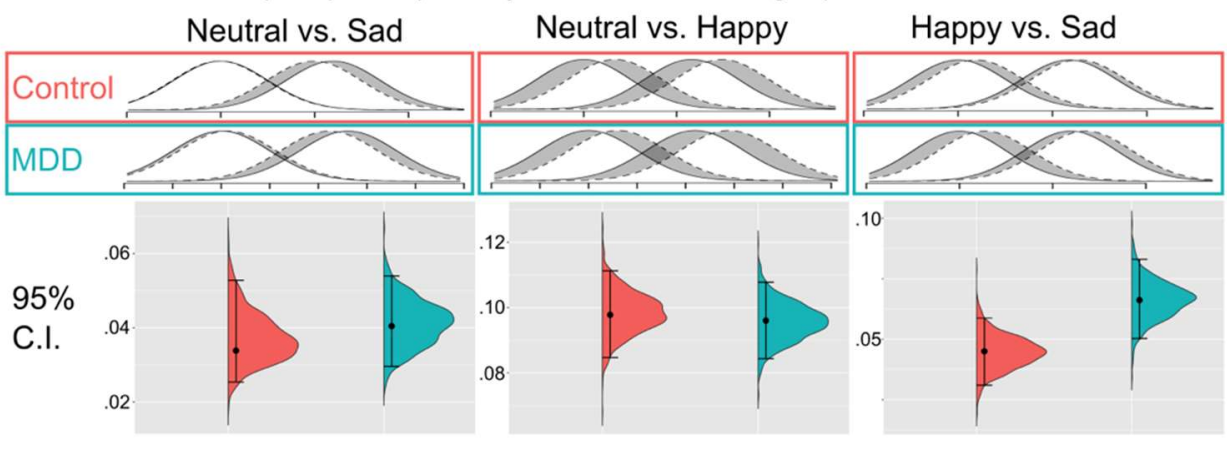

Does the result depend on specific face identities?

NO. Results did not change when the two identities presented were changed.

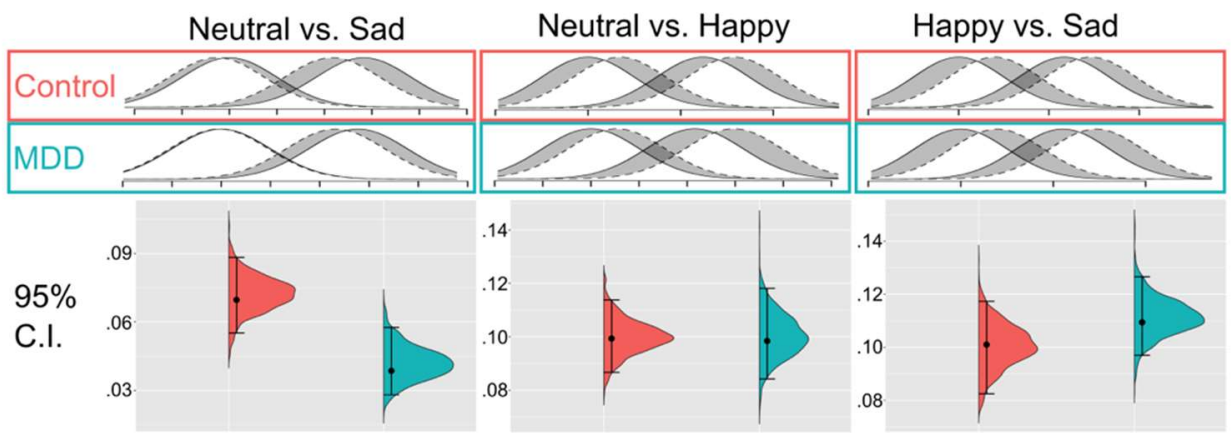

Does the result depend on ambiguity in the face information?

NO. Results did not change when ambiguity was increased.

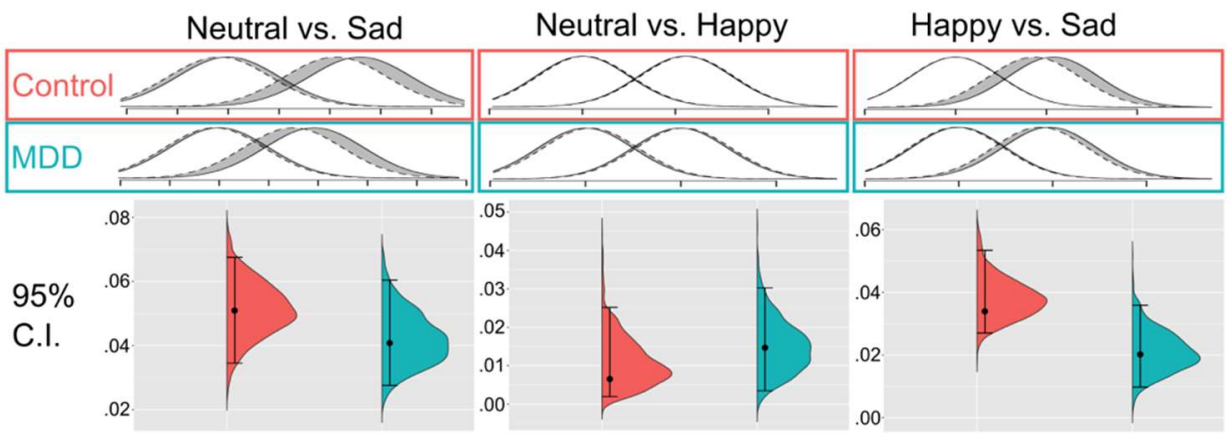

Figure S7. Detailed results on the analysis of perceptual separability of identity. Perceptual distributions recovered from the model are shown in the top row of each panel, with solid curves representing one emotional expression and dotted curves the other expression. The shaded area represents the L1 distance between perceptual distributions. 
Does MDD influence perceptual independence of emotion and identity?

NO. Deviations from perceptual independence were similar between groups for all emotions.

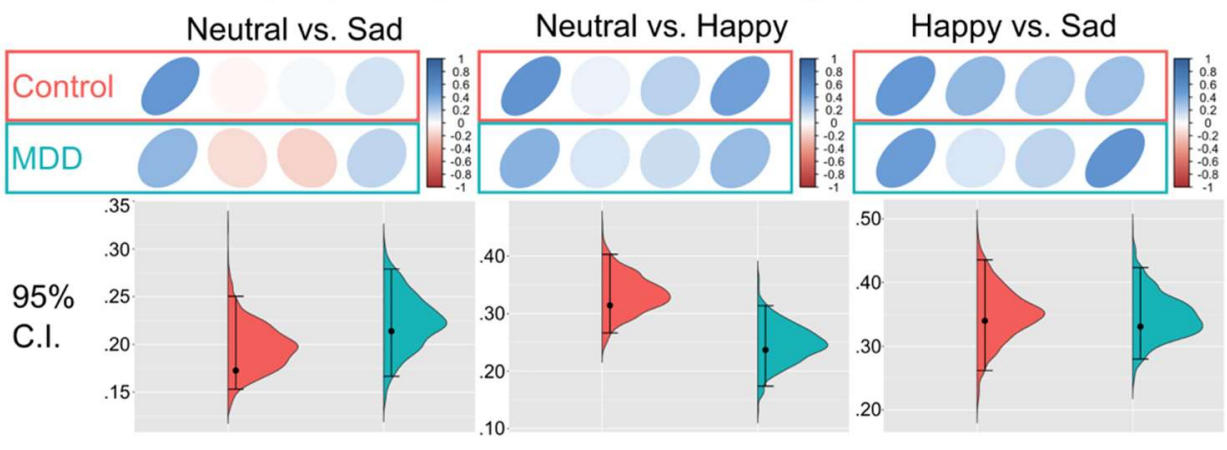

Does the result depend on specific face identities?

ONLY FOR HAPPINESS, where deviations from independence were stronger for MDD.

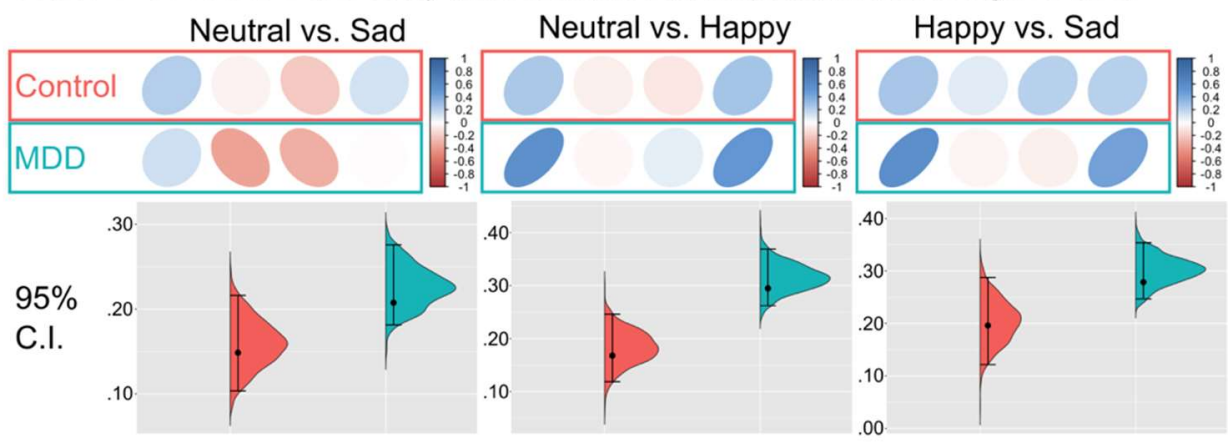

Does the result depend on ambiguity in the face information?

NO. Results did not change when ambiguity was increased.

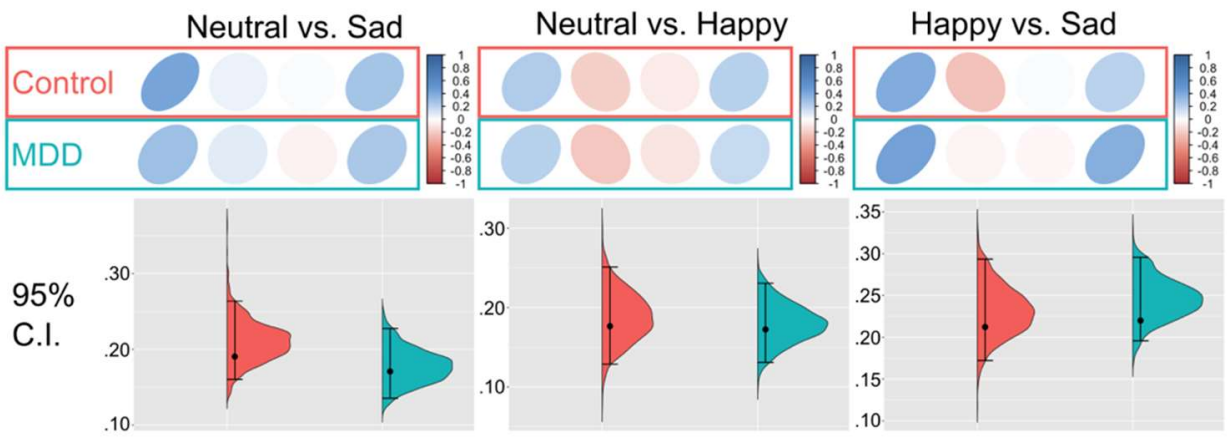

Figure S8. Results on the analysis of perceptual independence. The ellipses represent recovered correlation values for each stimulus. Stronger correlations are represented with more elongated ellipses with a stronger hue. The y-axis in the CI plots represents the mean absolute value of the correlations, with higher values representing stronger deviations from perceptual independence. The wealth of the evidence suggests that perceptual independence was not influenced by MDD. 
Does MDD influence the threshold bias for emotion?

NO. No differences in thresholds were found between groups,

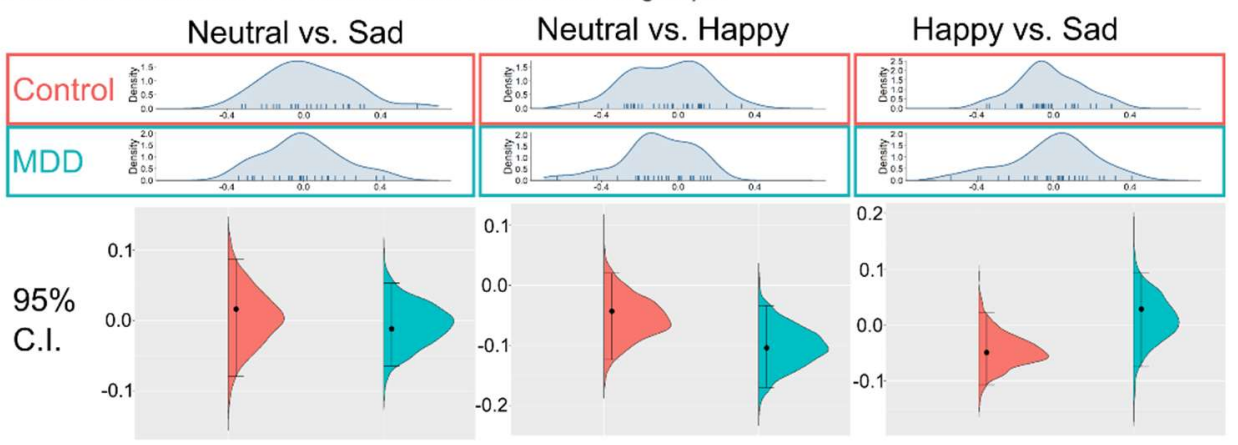

Does the result depend on specific face identities?

NO. Results did not change when the two identities presented were changed.

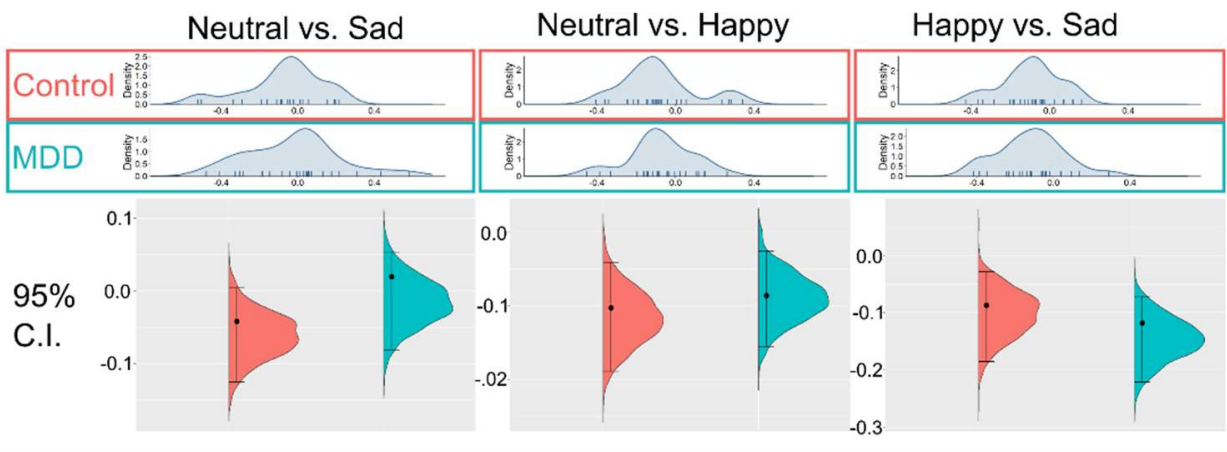

Does the result depend on ambiguity in the face information?

NO. Results did not change when ambiguity was increased in the stimuli.

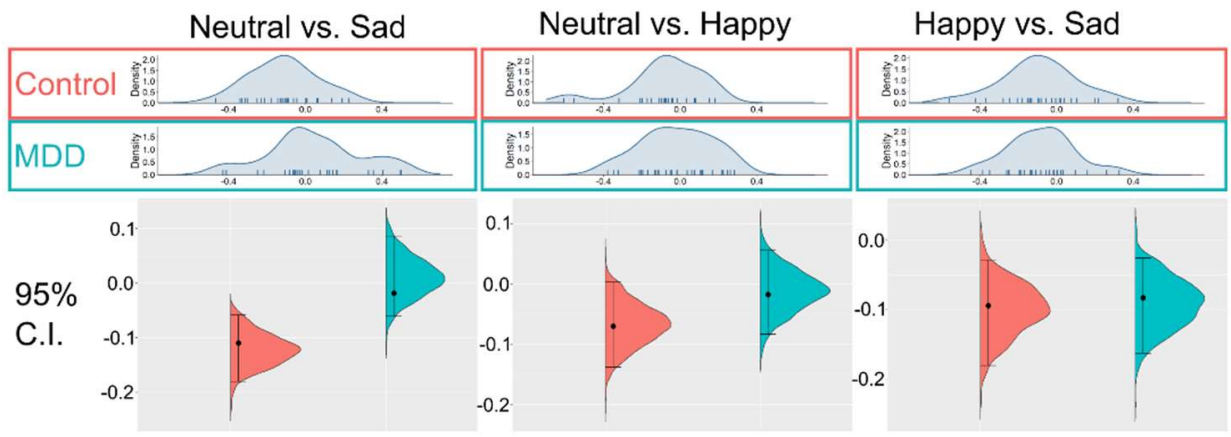

Figure S9. Detailed results on the analysis of threshold bias of emotion. The blue distributions at the top of each panel represent the distribution of bias values (tick marks represent individual values). The y-axis in the CI plots represents median bias. See main manuscript for more details. 
Does MDD influence the decisional separability of emotion?

YES. Deviations from decisional separability were different between groups for some emotion pairs.

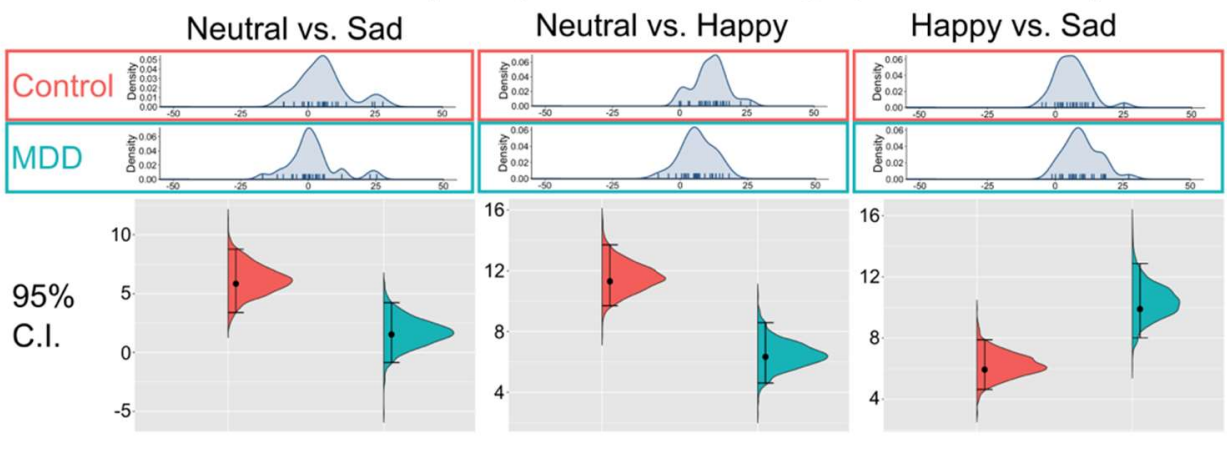

Does the result depend on specific face identities?

YES. Differences were found again, but the results pattern changed when the identities were changed.

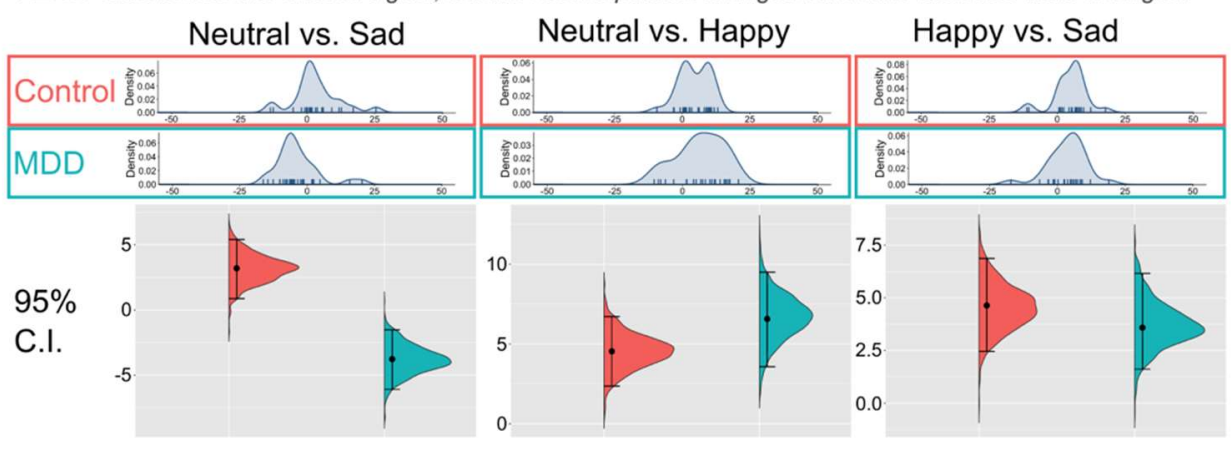

Does the result depend on ambiguity in the face information?

YES. No differences between groups were found when ambiguity was increased.

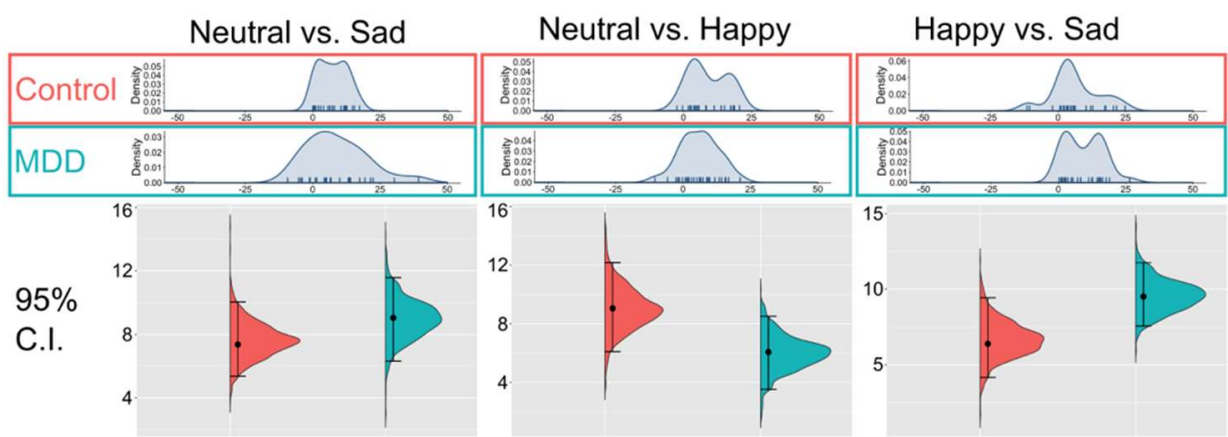

Figure S10. Results on the analysis of decisional separability of emotion. The blue distributions at the top of each panel represent angle of clockwise rotation of the decision bounds (tick marks represent individual values). Here, any deviation from zero (negative or positive) represents a violation of decisional separability. The y-axis in the CI plots represents mean clockwise rotation of the decision bounds. MDD does influence decisional separability of emotion but, as expected from decisional strategies, which are flexible and depend on the context of the task and stimuli, the pattern of results is highly variable and complex. MDD shows stronger deviations from decisional separability (i.e., rotation of bounds farther away from zero) in some cases (top panel: happy vs. sad; middle panel: neutral vs. sad) and weaker deviations in other cases (top panel: neutral vs. happy). While the results strongly support our suggestion that the influence of decisional strategies must be dissociated from perceptual effects, the strategies displayed by participants seem difficult to interpret. 
Does MDD influence decisional separability of identity?

YES. Deviations from decisional separability were different between groups for some emotion pairs.

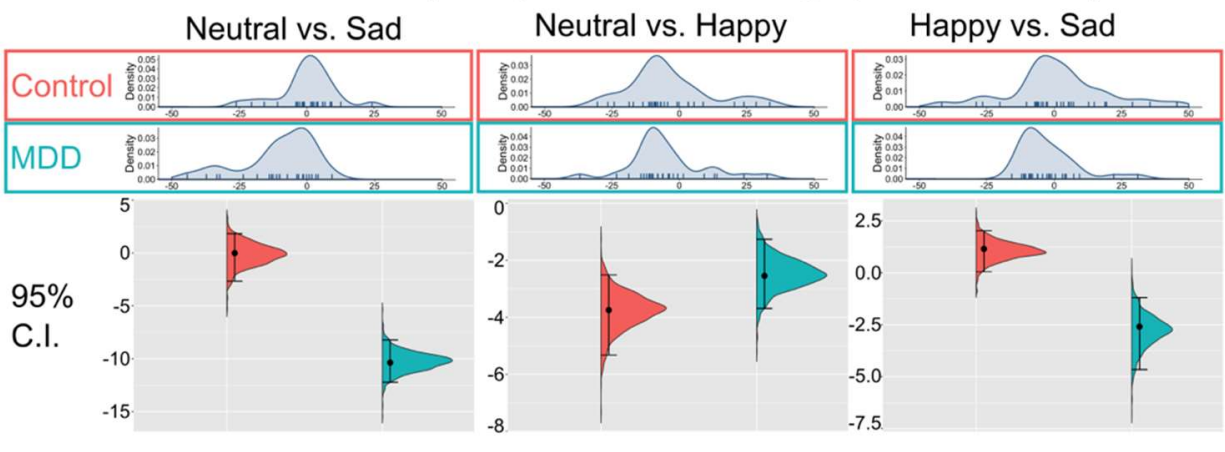

Does the result depend on specific face identities?

YES. Differences between groups were not found when the identities were changed.

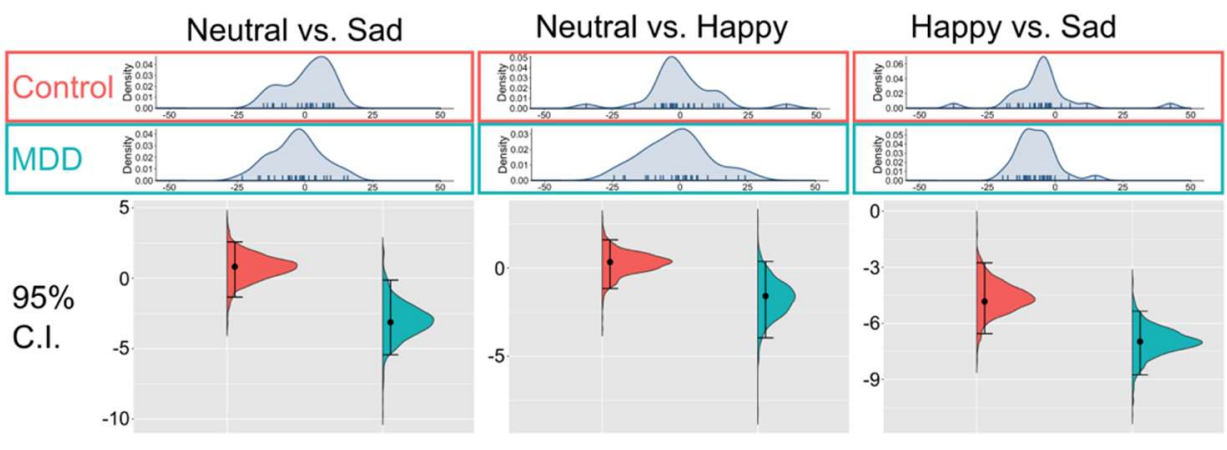

Does the result depend on ambiguity in the face information?

YES. The pattern of differences between groups changed when ambiguity was increased.

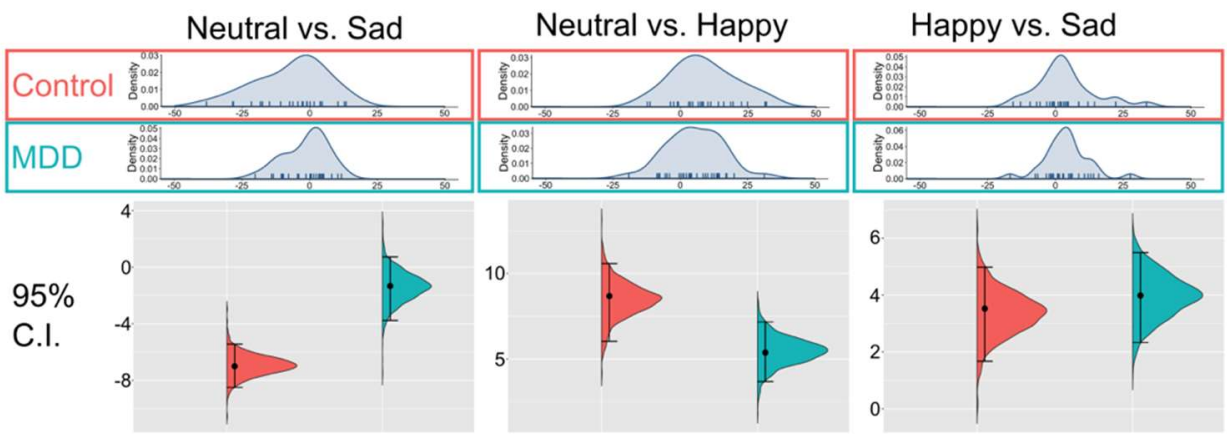

Figure S11. Results on the analysis of decisional separability of identity. The blue distributions at the top of each panel represent angle of clockwise rotation of the decision bounds (tick marks represent individual values). Here, any deviation from zero (negative or positive) represents a violation of decisional separability. The y-axis in the CI plots represents mean clockwise rotation of the decision bounds. MDD does influence decisional separability of emotion but, as expected from decisional strategies, which are flexible and depend on the context of the task and stimuli, the pattern of results is highly variable and complex. MDD shows stronger deviations from decisional separability (i.e., rotation of bounds farther away from zero) in some cases (top panel: neutral vs. sad, happy vs. sad) and weaker deviations in other cases (bottom panel: neutral vs. sad). While the results strongly support our suggestion that the influence of decisional strategies must be dissociated from perceptual effects, the strategies displayed by participants seem difficult to interpret. 


\section{Parameter recoverability in GRT-wIND}

We performed two simulations to evaluate the ability of our procedures to recover GRTwIND parameters when the ground truth is known. This serves to purposes. First, it allows to show the identifiability of parameters in the GRT-wIND model fitted to data in the main analysis. If two or more parameters in the model are not identifiable, then it would be difficult to recover the values of those parameters. Second, it allows to understand the precision of our maximum likelihood procedure to recover different parameters with the kind of data that we observed.

In both simulations, we took the 18 models estimated from data in the main analysis and we sampled data from them. We made sure to sample the exact same number of participants as in the original experiment, as well as the same number of data points per row in the confusion matrix of each participant, to obtain data with the same features and limitations as our original data (i.e., sampling more data might lead to better parameter recovery than what was possible in our study).

In the first simulation, we sampled data from the estimated models and then used the simulated data to fit a GRT-wIND model using the same procedures as in the main analysis, detailed above. We then sorted parameters of the original and recovered model into eight groups: means, correlations, lambdas, kappas, slopes of emotion bounds, position of emotion bounds, slopes of identity bounds, and position of identity bounds. We computed the Pearson correlation between the vectors of original and recovered parameters, as well as their $95 \%$ confidence intervals. We also tested whether the correlations were significantly higher than zero.
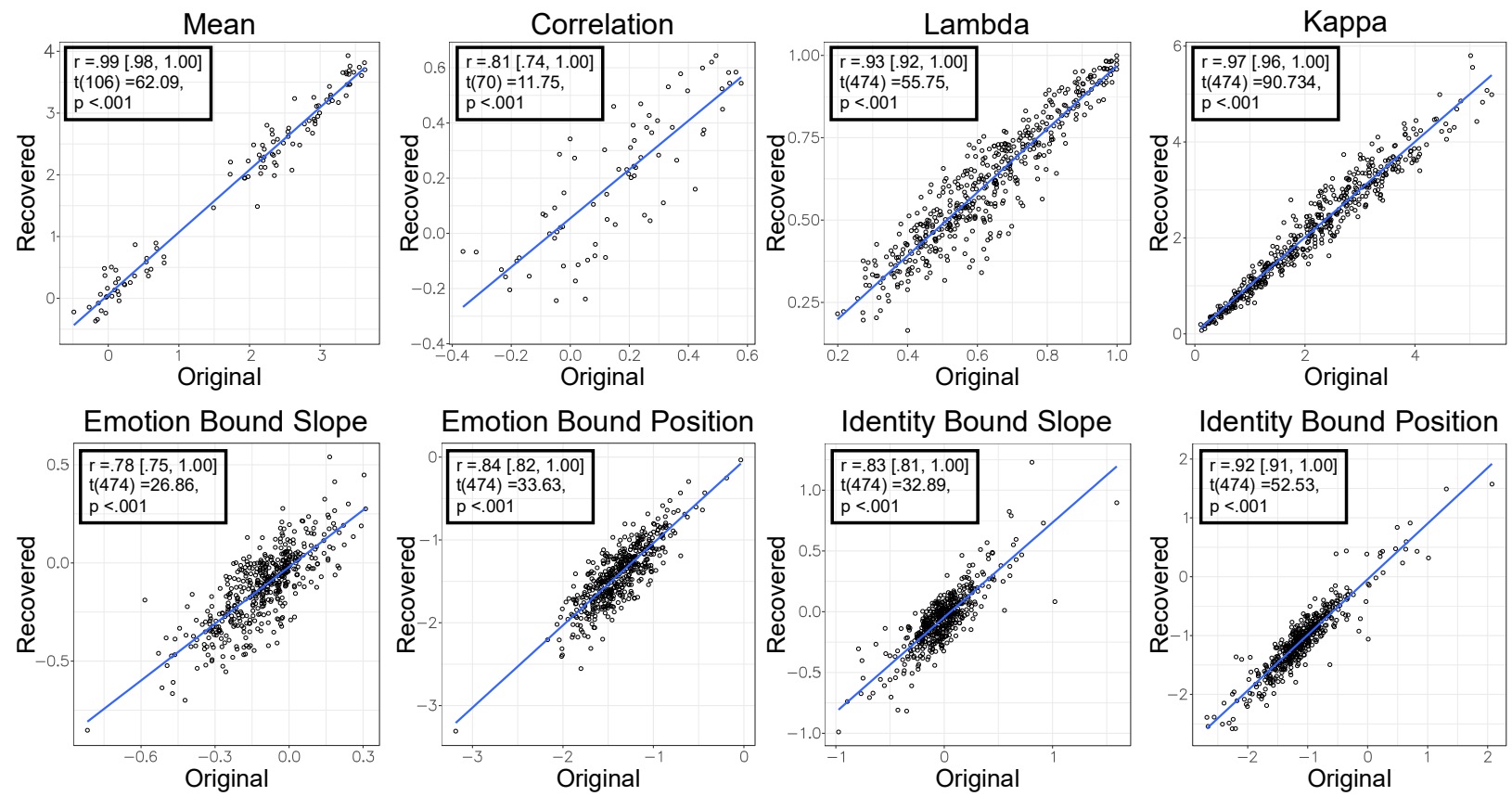

Figure S12. Scatterplots showing the relation between original and recovered GRT-wIND parameters in simulation 1. Blue lines represent linear regression models, with grey bands representing its $95 \%$ confidence interval. The inserts include information about the observed correlation, its 95\% confidence interval (within brackets), and test of significance.

Results are shown in Figure S12. It can be seen that the original and recovered parameters were highly correlated. To put the obtained correlation values in context, they were 
as high or higher than those obtained in simulation work with sequential sampling models of decision making (van Ravenzwaaij \& Oberauer, 2009), under ideal conditions in which large numbers of trials were obtained for each condition. Such models are widely used in cognitive psychology, computational psychiatry, and neuroscience research. The high correlations observed in Figure S9 make it unlikely that there are any identifiability problems with the parameters in GRT-wIND. In addition, the results suggest that parameter recoverability should be very good in our study.

In the second simulation, we investigated to what extent our modeling approach can truly disentangle perceptual from decisional factors influencing performance in an identification task. For this, we started by taking each of the parameters determining decision bounds (slopes of emotion bounds, position of emotion bounds, slopes of identity bounds, and position of identity bounds) and pooling them across all 18 estimated models from the main analysis. We estimated the distribution of parameters across participants and models using kernel density estimation. These distribution estimates allowed us to randomly re-sample decision bound parameters without creating decision bounds that are implausible. For each participant, we re-sampled each decision bound parameter independently from all others. This re-sampling resulted in a new set of 18 models, which had the same parameters defining perceptual factors as in the originally estimated models (means and correlations of perceptual distributions, plus attentional parameters kappa and lambda), but had completely different decisional strategies for each participant in each study. The question, then, was whether it would be possible to recover the original perceptual parameters using data obtained from models with completely different decisional strategies as the original models.
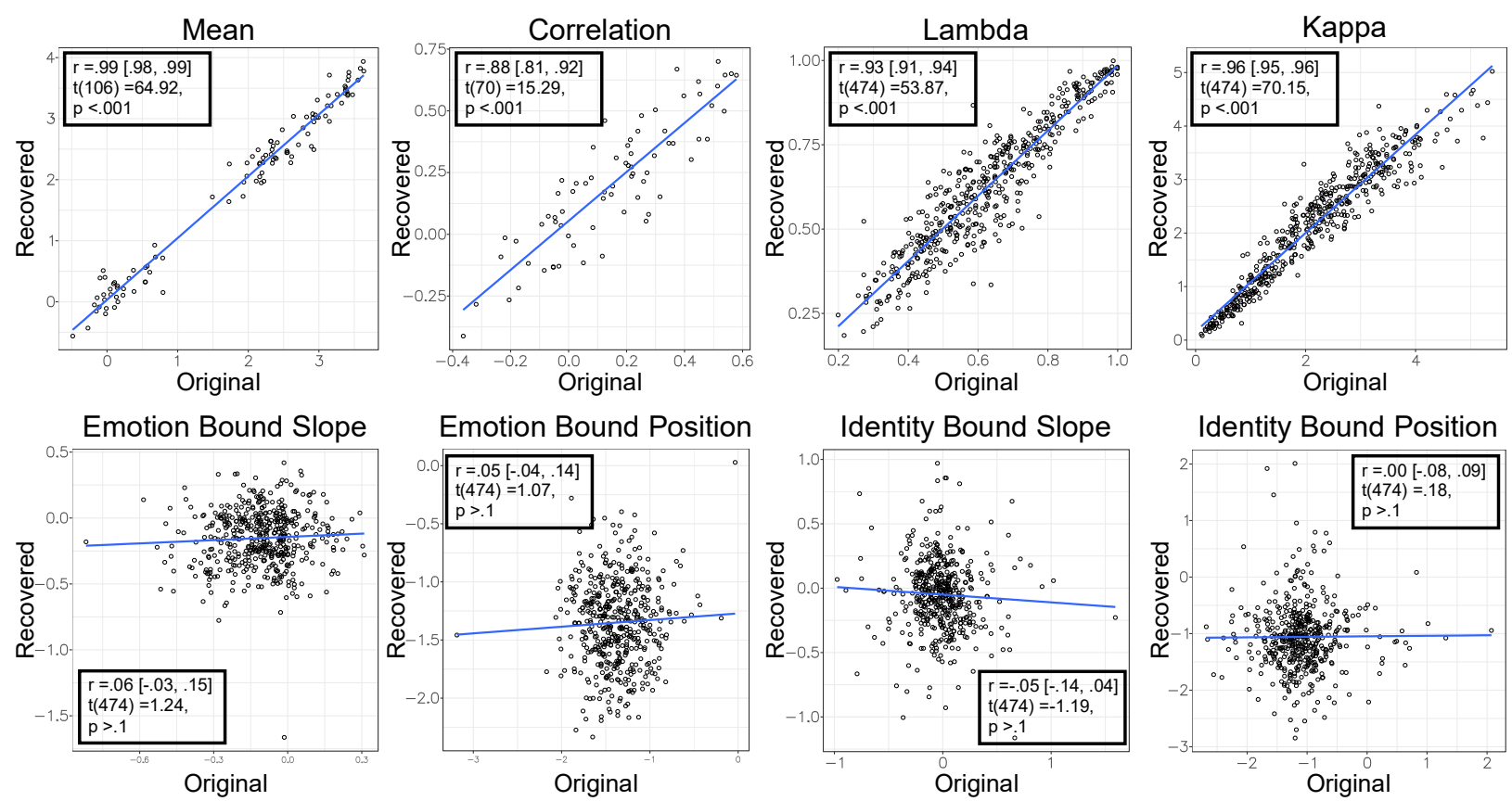

Figure S13. Scatterplots showing the relation between original and recovered GRT-wIND parameters in simulation 2. Blue lines represent linear regression models, with grey bands representing its $95 \%$ confidence interval. The inserts include information about the observed correlation, its 95\% confidence interval (within brackets), and test of significance. 
Results are shown in Figure S13. The top row of plots shows that the original and recovered perceptual parameters were again highly correlated, indicating that it is possible to recover perceptual parameters regardless of what decisional strategies are shown by the participants. That is, our modeling approach can truly disentangle perceptual from decisional factors influencing performance in an identification task.

The bottom row of plots in Figure S13 shows the correlations between decisional parameters in the original models and those recovered. These plots simply serve to show that our random sampling of decisional strategies did indeed change the decisional parameters in the model across participants. All correlations are close to zero and non-significant.

\section{References}

Ashby, F. G., Maddox, W. T., \& Lee, W. W. (1994). On the dangers of averaging across subjects when using multidimensional scaling or the similarity-choice model. Psychological Science, 5(3), 144-151.

Carpenter, J., \& Bithell, J. (2000). Bootstrap confidence intervals: when, which, what? A practical guide for medical statisticians. Statistics in Medicine, 19(9), 1141-1164.

Deneve, S., Latham, P. E., \& Pouget, A. (1999). Reading population codes: a neural implementation of ideal observers. Nature Neuroscience, 2(8), 740-745. https://doi.org/10.1038/11205

Good, P. I. (2006). Resampling methods: a practical guide to data analysis. Boston, MA: Birkhäuser.

Hamilton, M. (1967). Development of a rating scale for primary depressive illness. The British Journal of Social and Clinical Psychology, 6(4), 278-296. https://doi.org/10.1111/j.20448260.1967.tb00530.x

Lesmes, L. A., Lu, Z. L., Baek, J., Tran, N., Dosher, B. A., \& Albright, T. D. (2015). Developing Bayesian adaptive methods for estimating sensitivity thresholds ( $\left.d^{\prime}\right)$ in Yes-No and forced-choice tasks. Frontiers in Psychology, 6, 1070.

Linares, D., \& López-Moliner, J. (2016). quickpsy: An R package to fit psychometric functions for multiple groups. The R Journal, $8(1)$. Retrieved from http://diposit.ub.edu/dspace/handle/2445/116040

May, K. A., \& Solomon, J. A. (2015). Connecting psychophysical performance to neuronal response properties I: Discrimination of suprathreshold stimuli. Journal of Vision, 15(6), 8-8. https://doi.org/10.1167/15.6.8

Paradiso, M. A. (1988). A theory for the use of visual orientation information which exploits the columnar structure of striate cortex. Biological Cybernetics, 58(1), 35-49.

Rush, A. J., Trivedi, M. H., Carmody, T. J., Ibrahim, H. M., Markowitz, J. C., Keitner, G. I., Kornstein, S. G., Arnow, B., Klein, D. N., Manber, R., Dunner, D. L., Gelenberg, A. J., Kocsis, J. H., Nemeroff, C. B., Fawcett, J., Thase, M. E., Russell, J. M., Jody, D. N., Borian, F. E., \& Keller, M. B. (2005). Self-reported depressive symptom measures: sensitivity to detecting change in a randomized, controlled trial of chronically depressed, nonpsychotic outpatients. Neuropsychopharmacology: Official Publication of the American College of Neuropsychopharmacology, 30(2), 405-416. https://doi.org/10.1038/sj.npp.1300614 
Rush, A. J., Trivedi, M. H., Ibrahim, H. M., Carmody, T. J., Arnow, B., Klein, D. N., Markowitz, J. C., Ninan, P. T., Kornstein, S., Manber, R., Thase, M. E., Kocsis, J. H., \& Keller, M. B. (2003). The 16-Item Quick Inventory of Depressive Symptomatology (QIDS), clinician rating (QIDS-C), and self-report (QIDS-SR): a psychometric evaluation in patients with chronic major depression. Biological Psychiatry, 54(5), 573-583. https://doi.org/10.1016/s0006-3223(02)01866-8

Rush, A. J., Trivedi, M. H., Wisniewski, S. R., Nierenberg, A. A., Stewart, J. W., Warden, D., ... Lebowitz, B. D. (2006). Acute and longer-term outcomes in depressed outpatients requiring one or several treatment steps: a STAR* D report. American Journal of Psychiatry, 163(11), 1905-1917.

Series, P., Stocker, A. A., \& Simoncelli, E. P. (2009). Is the homunculus "aware" of sensory adaptation? Neural Computation, 21(12), 3271-3304.

Silbert, N. H., \& Thomas, R. (2013). Decisional separability, model identification, and statistical inference in the general recognition theory framework. Psychonomic Bulletin \& Review, 20(1), 1-20. https://doi.org/10.3758/s13423-012-0329-4

Silbert, N. H., \& Thomas, R. D. (2017). Identifiability and testability in GRT with individual differences. Journal of Mathematical Psychology, 77, 187-196. https://doi.org/10.1016/j.jmp.2016.08.002

Soto, F. A., Vucovich, L., Musgrave, R., \& Ashby, F. G. (2015). General recognition theory with individual differences: A new method for examining perceptual and decisional interactions with an application to face perception. Psychonomic Bulletin \& Review, 22(1), 88-111. https://doi.org/10.3758/s13423-014-0661-y

Susilo, T., McKone, E., \& Edwards, M. (2010). What shape are the neural response functions underlying opponent coding in face space? A psychophysical investigation. Vision Research, 50(3), 300-314. https://doi.org/10.1016/j.visres.2009.11.016

Trivedi, M. H., Rush, A. J., Ibrahim, H. M., Carmody, T. J., Biggs, M. M., Suppes, T., Crismon, M. L., Shores-Wilson, K., Toprac, M. G., Dennehy, E. B., \& Others. (2004). The Inventory of Depressive Symptomatology, Clinician Rating (IDS-C) and Self-Report (IDS-SR), and the Quick Inventory of Depressive Symptomatology, Clinician Rating (QIDS-C) and Self-Report (QIDS-SR) in public sector patients with mood disorders: a psychometric evaluation. Psychological Medicine, 34(1), 73-82.

https://www.cambridge.org/core/journals/psychological-medicine/article/inventory-ofdepressive-symptomatology-clinician-rating-idsc-and-selfreport-idssr-and-the-quickinventory-of-depressive-symptomatology-clinician-rating-qidsc-and-selfreport-qidssr-inpublic-sector-patients-with-mood-disorders-a-psychometricevaluation/6297492448377EC776825B39ABF070B4

van Ravenzwaaij, D., \& Oberauer, K. (2009). How to use the diffusion model: Parameter recovery of three methods: EZ, fast-dm, and DMAT. Journal of Mathematical Psychology, 53(6), 463-473. 